\title{
Radio light curves and imaging of the helium nova V445 Puppis reveal seven years of synchrotron emission
}

\author{
M. M. Nyamai ${ }^{1 \star}$, L. Chomiuk ${ }^{2} \dagger$, V. A. R. M. Ribeiro ${ }^{3,4}$, P. A. Woudt ${ }^{1}$, J. Strader $^{2}$, and \\ K. V. Sokolovsky 2,5 \\ ${ }^{1}$ Department of Astronomy, University of Cape Town, Private Bag X3, Rondebosch 7701, South Africa \\ ${ }^{2}$ Department of Physics and Astronomy, Michigan State University, East Lansing, MI 48824, USA \\ ${ }^{3}$ Instituto de Telecomunicações, Campus Universitário de Santiago, 3810-193 Aveiro, Portugal \\ ${ }^{4}$ Departamento de Física, Universidade de Aveiro, Campus Universitário de Santiago, 3810-193 Aveiro, Portugal \\ ${ }^{5}$ Sternberg Astronomical Institute, Moscow State University, Universitetskii pr. 13, 119992 Moscow, Russia
}

Accepted XXX. Received YYY; in original form ZZZ

\begin{abstract}
V445 Puppis is the only helium nova observed to date; its eruption in late 2000 showed high velocities up to $8500 \mathrm{~km} \mathrm{~s}^{-1}$, and a remarkable bipolar morphology cinched by an equatorial dust disc. Here we present multi-frequency radio observations of V445 Pup obtained with the Very Large Array (VLA) spanning 1.5-43.3 GHz, and between 2001 January and 2008 March (days $\sim 89-2700$ after eruption). The radio light curve is dominated by synchrotron emission over these seven years, and shows four distinct radio flares. Resolved radio images obtained in the VLA's A configuration show that the synchrotron emission hugs the equatorial disc, and comparisons to near-IR images of the nova clearly demonstrate that it is the densest ejecta - not the fastest ejecta - that are the sites of the synchrotron emission in V445 Pup. The data are consistent with a model where the synchrotron emission is produced by a wind from the white dwarf impacting the dense equatorial disc, resulting in shocks and particle acceleration. The individual synchrotron flares may be associated with density enhancements in the equatorial disc and/or velocity variations in the wind from the white dwarf. This overall scenario is similar to a common picture of shock production in hydrogen-rich classical novae, but V445 Pup is remarkable in that these shocks persist for almost a decade, much longer than the weeks or months for which shocks are typically observed in classical novae.
\end{abstract}

Key words: binaries:close - helium novae, cataclysmic variables - stars : outflows radio continuum: individual (V445 Puppis)- white dwarfs

\section{INTRODUCTION}

Extensive accretion of helium-rich material onto a white dwarf (WD) from a helium companion star results in increasing density and temperature on the WD surface, eventually triggering a helium shell flash (known as a helium nova; Kato et al. 1989). Helium novae occur for mass accretion rates in the range $\sim 10^{-8}$ to $10^{-7} \mathrm{M}_{\odot}$ $\mathrm{yr}^{-1}$ (Woosley \& Kasen 2011; Piersanti et al. 2013). The thermonuclear runaway ejects the outer accreted layers analogous to hydrogen-rich classical novae (see e.g., Bode \& Evans 2008; Woudt \& Ribeiro 2014), although more mass is ejected in helium novae (Kato et al. 1989;

^ nymmir001@myuct.ac.za

$\dagger$ chomiuk@pa.msu.edu
Hillman et al. 2016). However, when the helium accretion rate is lower $\left(\leq 10^{-8} \mathrm{M}_{\odot} \mathrm{yr}^{-1}\right)$, it is possible for a helium ignition on the surface of the WD to trigger an inward shockwave which leads to an explosion at the core of the WD hence a double detonation (Nomoto 1982; Shen \& Bildsten 2009). If the detonation occurs on a carbon-oxygen WD, it could produce a Type Ia supernova (SN Ia; Moll \& Woosley 2013; Ruiter et al. 2014; Piersanti et al. 2014). The study of helium accretion is therefore important for validating or ruling out helium-donor and WD binary systems as progenitors of SNe Ia.

In this paper, we present radio observations of the only spectroscopically confirmed helium nova, V445 Puppis. The nova was discovered in eruption in late November 2000 at an optical maximum of $V \approx 8.6$ mag (Kato et al. 2000), sub- 
stantially brighter than its $V \approx 14.5$ pre-eruption magnitude (Ashok \& Banerjee 2003). The day of eruption for V445 Pup is unknown and only constrained to be between 2000 September 26 and 2000 November 28 (Ashok \& Banerjee 2001; Kato et al. 2000; Woudt et al. 2009). We adopt a $t_{0}$ from Woudt et al. 2009 of 2000 November $02(\mathrm{MJD}=51850)$ as the time of eruption. The nova was unusually rich in carbon and showed helium emission lines, but lacked hydrogen lines (which are prominent in the spectra of classical novae; Ashok \& Banerjee 2003; Iijima \& Nakanishi 2008; Woudt et al. 2009).

Within one month of the eruption, dust was detected from the nova using infrared spectroscopy (Lynch et al. 2004). By $\sim 8$ months after the nova eruption, the optical brightness had dropped below pre-eruption levels (Ashok \& Banerjee 2003; Woudt et al. 2009), attributed to a strong dust formation episode. Six years following the eruption, the optical light had still not returned to pre-eruption levels (Woudt et al. 2009), and the dust mass was determined to be $>10^{-5} \mathrm{M}_{\odot}$ (Shimamoto et al. 2017).

V445 Pup was spatially resolved using near-infrared adaptive optics imaging with the Very Large Telescope (VLT), revealing an expanding bipolar shell confined by an equatorial dust disc and with polar knots on both ends of the bipolar ejecta (Woudt et al. 2009; see their Figure 2). Woudt et al. (2009) determined a distance of $8.2 \pm 0.5 \mathrm{kpc}$ to the nova using expansion parallax techniques.

Presented in this paper are multifrequency observations of the nova obtained in the years following eruption using the Very Large Array (VLA) radio telescope. The radio data reveal an unprecedented, near decade-long, synchrotronpowered radio light curve. In $\S 2$, we discuss the radio observations and our data analysis procedure. In $\S 3$, we present the multi-frequency radio light curve, radio spectral evolution, and spatially-resolved radio imaging. In $\S 4$, we present the results, and in $\S 5$ we highlight our conclusions.

\section{OBSERVATIONS AND DATA ANALYSIS}

Radio observations of V445 Pup were obtained with a variety of VLA programs, most of which were led by M. Rupen. The data were retrieved from the VLA archive (see Table 1 for a $\log$ of the observations). The first radio observations of V445 Pup were taken on 2001 January 18 and $30\left(t-t_{0}\right.$ $=77$ and 89 days). On day 77 , the nova was not detected at $8.4 \mathrm{GHz}$ (Rupen et al. 2001), and there was a marginal detection on day 89. After day 89, observations were paused until 2001 September 9 (day 312 day), when the nova was strongly detected at $8.4 \mathrm{GHz}$ (Rupen et al. 2001). V445 Pup was subsequently observed with the VLA from September 2001 to March 2008 (between day 312-2704), resulting in a detailed radio light curve spanning almost a decade following the eruption (Figure 1).

Observations were obtained in continuum mode, at different frequency bands with two $50 \mathrm{MHz}$-wide frequency channels. The observations were conducted at $\mathrm{L}(1.46 \mathrm{GHz})$, $\mathrm{C}(4.86 \mathrm{GHz}), \mathrm{X}(8.46 \mathrm{GHz}), \mathrm{U}(14.94 \mathrm{GHz}), \mathrm{K}(22.46 \mathrm{GHz})$ and $\mathrm{Q}(43.34 \mathrm{GHz})$ bands, and are used to trace the nova emission throughout all of the VLA's configurations. The VLA A configuration provides the highest resolution (synthesized beam FWHM of $2^{\prime \prime}$ at $1.5 \mathrm{GHz}$ and $0.3^{\prime \prime}$ at $8.5 \mathrm{GHz}$ ), and has the potential to provide resolved images (see $\S 3.3$ ). At each frequency, observations of the target are obtained together with observations of gain calibrators (see Table 2). The most common gain calibrator used was 0804-278, for which we took the ICRS coordinates RA $=08 \mathrm{~h} 04 \mathrm{~m} 51.451 \mathrm{~s}$ and Dec $=-27^{\circ} 49^{\prime} 11.32^{\prime \prime}$. We note that a slightly different RA position was used by National Radio Astronomy Observatory (NRAO) for this calibrator in 2001-2002 (RA = $08 \mathrm{~h} 04 \mathrm{~m} 51.440 \mathrm{~s}$ ); we, therefore, shifted the data so that all observations take $\mathrm{RA}=08 \mathrm{~h} 04 \mathrm{~m} 51.451 \mathrm{~s}$ (this is particularly relevant for our imaging results; §3.3). Flux density calibrators $0137+331(3 \mathrm{C} 48), 0542+498(3 \mathrm{C} 147)$ or $1331+305$ (3C286) were observed to set the absolute flux density scale. Some observations were obtained without a flux density calibrator; in most cases, we do not include these observations in this work. For the ones included, we used a flux calibrator of an adjacent epoch to set the flux density of the secondary calibrator.

All data were processed using the Common Astronomy Software Applications (CASA; McMullin et al. 2007). The A0flagger algorithm (Offringa et al. 2012) was used to flag data corrupted by radio frequency interference. Standard calibration procedures were applied to each observation, and calibration solutions were applied to the target data before imaging. Starting in 2006, the VLA antennas were gradually upgraded to Jansky VLA capabilities, and we therefore found baseline-based calibration solutions using the flux calibrator.The CASA task clean was used for imaging, utilising Briggs weighting with a robust value of 1 . Self-calibration was not performed.

To measure the flux densities of V445 Pup, the CASA task imfit was used to fit a Gaussian to the nova in each resulting image. In most measurements the width of the Gaussian is allowed to vary, and the integrated flux density was recorded. However, in cases of low signal-to-noise, the size of the Gaussian was fixed to the size of the synthesized beam. The uncertainty in flux density includes the error from the Gaussian fit added in quadrature with estimates of the uncertainty on the absolute flux calibration (5\% of the flux for $1-10 \mathrm{GHz}$ and $10 \%$ of the flux for frequencies greater than $10 \mathrm{GHz}$ ). In epochs where there is a non-detection, the task imstat was used to determine the noise of the image. An upper limit on the flux density is determined as three times the image rms plus the value of the pixel at the target location.

The flux densities of V445 Pup for each frequency band are given in Table 3; the first ten epochs are presented in this manuscript and others are available in the electronic materials.

\section{RESULTS}

\subsection{Radio light curves}

Multi-frequency observations, spanning 1.5-43.3 GHz, reveal an unusual radio light curve for the eruption of V445 Pup between 2001-2008 presented in Figure 1. The radio light curve shows four distinct flares throughout its evolution.

In the earliest radio observation following the eruption of V445 Pup, around day 77, no radio emission was detected, with a $3 \sigma$ upper limit of $0.34 \mathrm{mJy}$ at $8.4 \mathrm{GHz}$. However, on day 89 , a $4 \sigma$ detection of 0.35 mJy was obtained at $8.4 \mathrm{GHz}$. 
Table 1. Log of VLA observations of V445 Pup.

\begin{tabular}{|c|c|c|c|c|c|c|c|c|c|}
\hline \multirow{2}{*}{$\begin{array}{l}\text { Observation } \\
\text { Date }\end{array}$} & \multirow{2}{*}{$\begin{array}{c}t \\
\text { (MJD) }\end{array}$} & \multirow{2}{*}{$\begin{array}{c}t-t_{0} \\
(\text { Days }) \\
\end{array}$} & \multirow[t]{2}{*}{ Configuration } & \multicolumn{6}{|c|}{ Observation time on target $(\min )$} \\
\hline & & & & $1.43 \mathrm{GHz}$ & $4.86 \mathrm{GHz}$ & $8.46 \mathrm{GHz}$ & $14.94 \mathrm{GHz}$ & $22.46 \mathrm{GHz}$ & $43.34 \mathrm{GHz}$ \\
\hline 2001 Jan 18 & 51927 & 77 & A & $\ldots$ & $\ldots$ & 3.7 & $\ldots$ & $\ldots$ & $\ldots$ \\
\hline 2001 Jan 30 & 51939 & 89 & A & $\ldots$ & $\ldots$ & 6.4 & $\ldots$ & $\ldots$ & $\ldots$ \\
\hline 2001 Sept 09 & 52162 & 312 & $\mathrm{C}$ & $\ldots$ & $\ldots$ & 6.7 & $\ldots$ & $\ldots$ & $\ldots$ \\
\hline 2001 Sept 11 & 52164 & 314 & $\mathrm{C}$ & 15.6 & 3.7 & $\ldots$ & 5.2 & 4.6 & 4.4 \\
\hline 2001 Sept 12 & 52165 & 315 & $\mathrm{C}$ & 10.9 & 3.9 & 3.7 & 5.2 & 4.4 & $\ldots$ \\
\hline 2001 Sept 14 & 52167 & 317 & $\mathrm{C}$ & $\ldots$ & 4.1 & $\ldots$ & $\ldots$ & $\ldots$ & $\ldots$ \\
\hline 2001 Sept 15 & 52168 & 318 & $\mathrm{C} \& \mathrm{D}$ & $\ldots$ & 1.9 & 3.1 & $\ldots$ & $\ldots$ & $\ldots$ \\
\hline 2001 Sept 16 & 52169 & 319 & $C \& D$ & 5.2 & 6.9 & 6.9 & 4.9 & $\ldots$ & $\ldots$ \\
\hline 2001 Sept 17 & 52170 & 320 & $C \& D$ & 10.4 & 11.2 & $\ldots$ & 11.9 & $\ldots$ & $\ldots$ \\
\hline 2001 Sept 20 & 52173 & 323 & $C \& D$ & 3.7 & 4.2 & 4.2 & 4.2 & 4.2 & $\ldots$ \\
\hline 2001 Sept 25 & 52178 & 328 & $\mathrm{C} \& \mathrm{D}$ & $\ldots$ & 11.7 & 12.7 & 14.8 & $\ldots$ & $\ldots$ \\
\hline 2001 Sept 26 & 52179 & 329 & $\mathrm{C} \& \mathrm{D}$ & $\ldots$ & 3.2 & 4.7 & 6.2 & 6.2 & $\ldots$ \\
\hline
\end{tabular}

'...' indicates no observations for this epoch at that frequency. Here, $t_{0}$ is taken as 2000 November 02 (MJD $\left.=51850\right)$. This table is continued in Appendix C Table C1.

Table 2. Log of gain calibrators used at different configurations and observing frequencies.

\begin{tabular}{|c|c|c|c|c|c|c|c|}
\hline \multirow{2}{*}{$\begin{array}{l}\text { Observation } \\
\text { Date range }\end{array}$} & \multirow[b]{2}{*}{ Configuration } & \multicolumn{6}{|c|}{ Gain calibrators } \\
\hline & & $1.43 \mathrm{GHz}$ & $4.86 \mathrm{GHz}$ & $8.46 \mathrm{GHz}$ & $14.94 \mathrm{GHz}$ & $22.46 \mathrm{GHz}$ & $43.34 \mathrm{GHz}$ \\
\hline 2001 Jan 18-2001 Jan 30 & $\mathrm{~A}$ & $\ldots$ & $\ldots$ & $0804-278$ & $\ldots$ & $\ldots$ & $\ldots$ \\
\hline 2001 Sep 09-2001 Sep 14 & $\mathrm{C}$ & 0738-304 & $0804-278$ & $0804-278$ & 0804-278 & 0804-278 & 0804-278 \\
\hline 2001 Sep 15-2001 Oct 10 & $\mathrm{C} \& \mathrm{D}$ & $\begin{array}{l}0735-175 \\
0806-268 \\
0706-231\end{array}$ & $\begin{array}{l}0804-278 \\
0738-304\end{array}$ & $0804-278$ & $0804-278$ & $0804-278$ & 0804-278 \\
\hline 2001 Oct $12-2002$ Jan 13 & $\mathrm{D}$ & $\begin{array}{l}0706-231 \\
0806-268\end{array}$ & $\begin{array}{c}0738-304, \\
0804-278\end{array}$ & $0804-278$ & $0804-278$ & $0804-278$ & $0804-278$ \\
\hline 2002 Feb 07-2002 Jun 07 & $\mathrm{~A}, \mathrm{~A} \& \mathrm{~B}$ & $0804-278$ & $0804-278$ & $0804-278$ & $0804-278$ & 0804-278 & $\ldots$ \\
\hline 2002 Jun $17-2002$ Oct 31 & B & 0804-278 & 0804-278 & 0804-278 & $\ldots$ & $\ldots$ & 0804-278 \\
\hline 2002 Dec 09-2003 Jan 29 & $\mathrm{C}, \mathrm{C} \& \mathrm{D}$ & $\ldots$ & 0804-278 & 0804-278 & $\ldots$ & $\ldots$ & $\ldots$ \\
\hline 2003 Feb 06-2003 Apr 18 & $\mathrm{D}$ & $\ldots$ & 0804-278 & $0804-278$ & $\ldots$ & $\ldots$ & $\ldots$ \\
\hline 2003 May $30-2003$ Oct 08 & $\mathrm{~A}, \mathrm{~A} \& \mathrm{~B}$ & $\begin{array}{l}0735-175 \\
0804-278\end{array}$ & $0804-278$ & $0804-278$ & $\cdots$ & $\ldots$ & $\ldots$ \\
\hline 2003 Oct $21-2004$ Mar 02 & $\mathrm{~B}, \mathrm{~B} \& \mathrm{C}$ & $0804-278$ & $0804-278$ & 0804-278 & 0804-278 & 0804-278 & 0804-278 \\
\hline 2003 Mar 10-2004 June 12 & $\mathrm{C}, \mathrm{C} \& \mathrm{D}$ & $\begin{array}{l}0738-304 \\
0706-231\end{array}$ & $0804-278$ & 0804-278 & $\ldots$ & $0804-278$ & $\ldots$ \\
\hline 2004 Jun 25- 2004 Aug 21 & $\mathrm{D}$ & $\ldots$ & $0804-278$ & $0804-278$ & $0804-278$ & $\ldots$ & $\ldots$ \\
\hline 2004 Sep 09- 2005 Feb 03 & $\mathrm{~A}, \mathrm{~A} \& \mathrm{~B}$ & $\ldots$ & 0804-278 & 0804-278 & 0804-278 & $\ldots$ & $\ldots$ \\
\hline 2005 Feb 26-2005 Jul 02 & $\mathrm{~B}, \mathrm{~B} \& \mathrm{C}$ & $0804-278$ & 0804-278 & 0804-278 & $\ldots$ & $\ldots$ & $\ldots$ \\
\hline 2005 Jul $09-2005$ Oct 31 & $\mathrm{C}, \mathrm{C} \& \mathrm{D}$ & $\ldots$ & $0804-278$ & 0804-278 & $\ldots$ & $0804-278$ & $\ldots$ \\
\hline 2005 Nov 08-2006 Jan 22 & $\mathrm{D}$ & $\ldots$ & 0738-304 & 0804-278 & $\ldots$ & $\ldots$ & $\ldots$ \\
\hline 2006 Mar 15-2006 May 10 & $\mathrm{~A}$ & $\ldots$ & $\ldots$ & $0804-278$ & $\ldots$ & $\ldots$ & $\ldots$ \\
\hline 2006 Jun 25-2006 Sep 11 & B & $\ldots$ & 0804-278 & $0804-278$ & $\ldots$ & $\ldots$ & $\ldots$ \\
\hline 2007 Sep 30-2008 Jan 17 & $\mathrm{~A} \& \mathrm{~B}, \mathrm{~B}$ & $\ldots$ & $\ldots$ & 0804-278 & $\ldots$ & $\ldots$ & $\ldots$ \\
\hline 2007 Jan 23, 2008 Mar 28 & $\mathrm{C} \& \mathrm{D}, \mathrm{D}$ & $\ldots$ & $\ldots$ & 0804-278 & $\ldots$ & $\ldots$ & $\ldots$ \\
\hline
\end{tabular}

'...' indicates no observations at that date range and frequency.

Unfortunately, following the day 89 detection there were no radio observations of the nova until 312 days after eruption. As a result of very limited coverage during the first 300 days after eruption, these early points are not shown in Figure 1. On day 312, it became clear that V445 Pup had brightened at radio frequencies since day 89 by a factor of $\sim 26$ to 9.4 mJy at $8.4 \mathrm{GHz}$. Over the next $\sim 3$ months (see Figure 2), the light curve shows flux densities declining from a radio maximum, which presumably occurred before day 312 .

Other flares are superimposed on the decline, like the steep peak on day 337 (at 4.9/8.5/14.9 GHz; Figure 2). Another flare peaked between days 450 to 460 (at 4.86/8.46/14.94 GHz bands). We note that, for the lowest frequency $(1.43 \mathrm{GHz})$ light curve, these peaks occur later, at days 341 and 493 (Figure 2).
The radio peaks observed between day 300-700, indicated by vertical dashed lines in Figure 2, have sharp, rapid rises and falls in flux density, compared to the last broad peak which occurs between days 700 and 3000 (Figure 1). Furthermore, the flux density at the peak of the last flare is a factor of five fainter than the early-time peaks.

The last recorded flux density of the nova with the VLA was $0.19 \mathrm{mJy}$ at $8.46 \mathrm{GHz}$ on 2008 March 29 (day 2704). On 2009 October 29 and December 29 (days 3283 and 3325 respectively), V445 Pup was observed using the Giant Metrewave Radio Telescope (GMRT) at $1.28 \mathrm{GHz}$ and $1.40 \mathrm{GHz}$ (Kantharia 2012). Radio emission was present in the first GMRT observation, with a flux density $\sim 0.3 \pm 0.08 \mathrm{mJy}$. The second epoch yields a non-detection with a $3 \sigma$ upper limit of $60 \mu \mathrm{Jy}$ (Kantharia 2012). 
Table 3. Flux densities and spectral indices of V445 Pup

\begin{tabular}{|c|c|c|c|c|c|c|c|c|}
\hline \multirow{2}{*}{$\begin{array}{l}t \\
(\mathrm{MJD})\end{array}$} & \multirow{2}{*}{$\begin{array}{c}t-t_{0} \\
\text { (Days) }\end{array}$} & \multirow[b]{2}{*}{$1.43 \mathrm{GHz}$} & \multicolumn{3}{|c|}{ Radio flux densities (mJy) } & \multirow[b]{2}{*}{$22.46 \mathrm{GHz}$} & \multirow[b]{2}{*}{$43.34 \mathrm{GHz}$} & \multirow[t]{2}{*}{$\alpha$} \\
\hline & & & $4.86 \mathrm{GHz}$ & $8.46 \mathrm{GHz}$ & $14.94 \mathrm{GHz}$ & & & \\
\hline 51927 & 77 & $\ldots$ & $\ldots$ & $<0.34$ & $\ldots$ & $\ldots$ & $\ldots$ & $\ldots$ \\
\hline 51939 & 89 & $\ldots$ & $\ldots$ & $0.354 \pm 0.064$ & $\ldots$ & $\ldots$ & $\ldots$ & $\ldots$ \\
\hline 52162 & 312 & $\ldots$ & $\ldots$ & $9.39 \pm 0.54$ & $\ldots$ & $\ldots$ & $\ldots$ & $\ldots$ \\
\hline 52164 & 314 & $21.27 \pm 1.22$ & $12.66 \pm 0.71$ & $\ldots$ & $4.83 \pm 0.82$ & $3.71 \pm 0.50$ & $3.87 \pm 1.03$ & $-0.57 \pm 0.08$ \\
\hline 52165 & 315 & $20.47 \pm 1.24$ & $11.59 \pm 0.64$ & $8.00 \pm 0.54$ & $5.96 \pm 0.90$ & $3.29 \pm 0.72$ & $\ldots$ & $-0.54 \pm 0.05$ \\
\hline 52167 & 317 & $\ldots$ & $11.18 \pm 0.62$ & $\ldots$ & $\ldots$ & $\ldots$ & $\ldots$ & $\ldots$ \\
\hline 52168 & 318 & $\ldots$ & $9.99 \pm 0.66$ & $6.77 \pm 0.45$ & $\ldots$ & $\ldots$ & $\ldots$ & $\ldots$ \\
\hline 52169 & 319 & $18.86 \pm 1.24$ & $9.52 \pm 0.55$ & $5.74 \pm 0.38$ & $4.54 \pm 0.57$ & $\ldots$ & $\ldots$ & $-0.64 \pm 0.06$ \\
\hline 52170 & 320 & $17.55 \pm 1.26$ & $9.30 \pm 0.50$ & $6.03 \pm 0.41$ & $4.48 \pm 0.57$ & $2.62 \pm 0.62$ & $\ldots$ & $-0.61 \pm 0.05$ \\
\hline 52173 & 323 & $17.25 \pm 1.07$ & $9.07 \pm 0.62$ & $6.48 \pm 0.76$ & $4.90 \pm 0.72$ & $4.44 \pm 0.79$ & $\ldots$ & $-0.52 \pm 0.02$ \\
\hline 52178 & 328 & $11.71 \pm 0.82$ & $8.44 \pm 0.45$ & $7.40 \pm 0.39$ & $5.46 \pm 0.69$ & $\ldots$ & $\ldots$ & $-0.28 \pm 0.03$ \\
\hline 52179 & 329 & $\ldots$ & $8.89 \pm 0.48$ & $8.50 \pm 0.46$ & $6.58 \pm 0.73$ & $4.63 \pm 0.64$ & $\ldots$ & $-0.33 \pm 0.13$ \\
\hline
\end{tabular}

'...' indicates no measurements for flux density for the epoch at that frequency. This table is continued in Appendix C Table C2.

\subsection{Radio spectral evolution}

We determine the spectral index for each observation $(\alpha$, defined as $S_{v} \propto v^{\alpha}$, where $S_{v}$ is the flux density and $v$ is the observing frequency), from the slope of the line fit to the data in log-log scale. The python function curve-fit in the scipy package is used to perform the least-squares fit. Detections separated by less than a day are combined into one spectral index fit. Selected spectra are plotted in Figure 3, while the lower panel of Figure 1 shows how the spectral index varies with time.

During the first decay of the radio flux density (days $\sim 312-400$ ), the spectrum has a form that rises steeply toward low frequencies and is well fit with a single power law. For example, on day 314, $\alpha=-0.6$ (top left panel of Figure 3 ), an indication of optically-thin synchrotron emission (see $§ 4.1$ for more discussion). Sometimes the spectrum appears to flatten toward low frequency (such as on day 337 ) and afterwards the spectrum switches back to being well fit with a single power law (e.g., day 383)

On days 429 and 447 , the spectrum becomes inverted and rises towards higher frequencies, with $\alpha \approx 0.4$ (Figure 3 ), an indication of optically-thick emission. On days 462 and 469 , the radio spectrum is transitioning back to an opticallythin state, exhibiting a combination of inverted/flat spectral index at lower frequencies and steep spectral index at higher frequencies. By day 493, the radio spectrum has returned to optically thin, hovering around $\alpha \approx-0.5$. We discuss likely causes of these changes in the radio spectrum in $\S 4.3$.

\subsection{Radio Images}

In its most extended A configuration, the VLA achieves angular resolution sufficient to constrain the morphology of radio emission from V445 Pup. The dates of these highresolution observations are listed in Table 4, along with a brief description of the resulting images and the central position of the emission region (more details below). We fit the morphology of V445 Pup in the $u v$-plane, using the UVFIT task in AIPS (Greisen 2003a) and Difmap (Shepherd et al. 1994; Shepherd 1997a).

The VLA was in A configuration during the first observations of V445 Pup in 2001 Jan. They yielded a nondetection (Jan $17, t-t_{0}=77$ days) and a marginal detec- tion (Jan $30, t-t_{0}=89$ days). The $\mathrm{S} / \mathrm{N}$ was not sufficient to constrain the morphology of V445 Pup at this time.

During the 2002 A configuration (462-559 days after eruption), V445 Pup was radio bright. At $8.4 \mathrm{GHz}$, it is unresolved; model fitting with an elliptical Gaussian in the $u v$ plane implies FWHM $<0.1^{\prime \prime}$.

During the 2003 A configuration (926-1051 days after eruption), V445 Pup is marginally resolved. We model the $8.4 \mathrm{GHz}$ data in the $u v$ plane with an elliptical Gaussian with a major axis of $0.25^{\prime \prime} \pm 0.04^{\prime \prime}$, unresolved minor axis $\left(<0.1^{\prime \prime}\right)$, and position angle of $92^{\circ} \pm 14^{\circ}$ (degrees east from north). Subtracting this Gaussian in the $u v$ plane and imaging the residuals, there is some structure remaining which implies that the source is not fully described as a single Gaussian. The central position of the 2003 emission is near the 2002 position, although not consistent within the formal errors (Table 4). Inspection of the images implies that they likely share a common position, but the more complex morphology of the 2003 image leads to a slight apparent offset.

During the first months of the 2004 A configuration (1408-1488 days after eruption), V445 Pup is resolved into two distinct regions of emission that are roughly aligned with the major axis of the 2003 elliptical Gaussian fit. In the $8.4 \mathrm{GHz}$ observations, the structure of V445 Pup can be fit with two circular Gaussian components separated by $0.22^{\prime \prime} \pm 0.02^{\prime \prime}$. No significant motion of the components could be seen over $\sim 4$ months of observations. The north-eastern (NE) component's ICRS coordinates are $\mathrm{RA}=7 \mathrm{~h} 37 \mathrm{~m} 56.890 \mathrm{~s}$, Dec $=-25^{\circ} 56^{\prime} 58.80^{\prime \prime}$, and the coordinates of the south-western (SW) component are RA = $7 \mathrm{~h} 37 \mathrm{~m} 56.875 \mathrm{~s}$, Dec $=-25^{\circ} 56^{\prime} 58.88^{\prime \prime}$. The components themselves are marginally resolved, with the NE component's $\mathrm{FWHM}=0.16^{\prime \prime} \pm 0.05^{\prime \prime}$ and the SW component measured to have a FWHM $=0.12^{\prime \prime} \pm 0.03^{\prime \prime}$. The 2004 components are located on either side of the radio emission imaged in $2002 / 2003$, consistent with them moving away from this origin position. The two 2004 components are similar in brightness, with flux densities of $0.7 \pm 0.1 \mathrm{mJy}(\mathrm{NE})$ and $0.5 \pm 0.1$ mJy (SW). The NE component gradually fades while the SW component gradually brightens and remains the only visible component by the end of 2004 .

Figure 4 compares a VLA image produced by stacking the $8.4 \mathrm{GHz}$ observations obtained during the $2004 \mathrm{~A}$ configuration with the near-IR high-resolution image, obtained by Woudt et al. (2009) on 2005 Mar 26 using the 


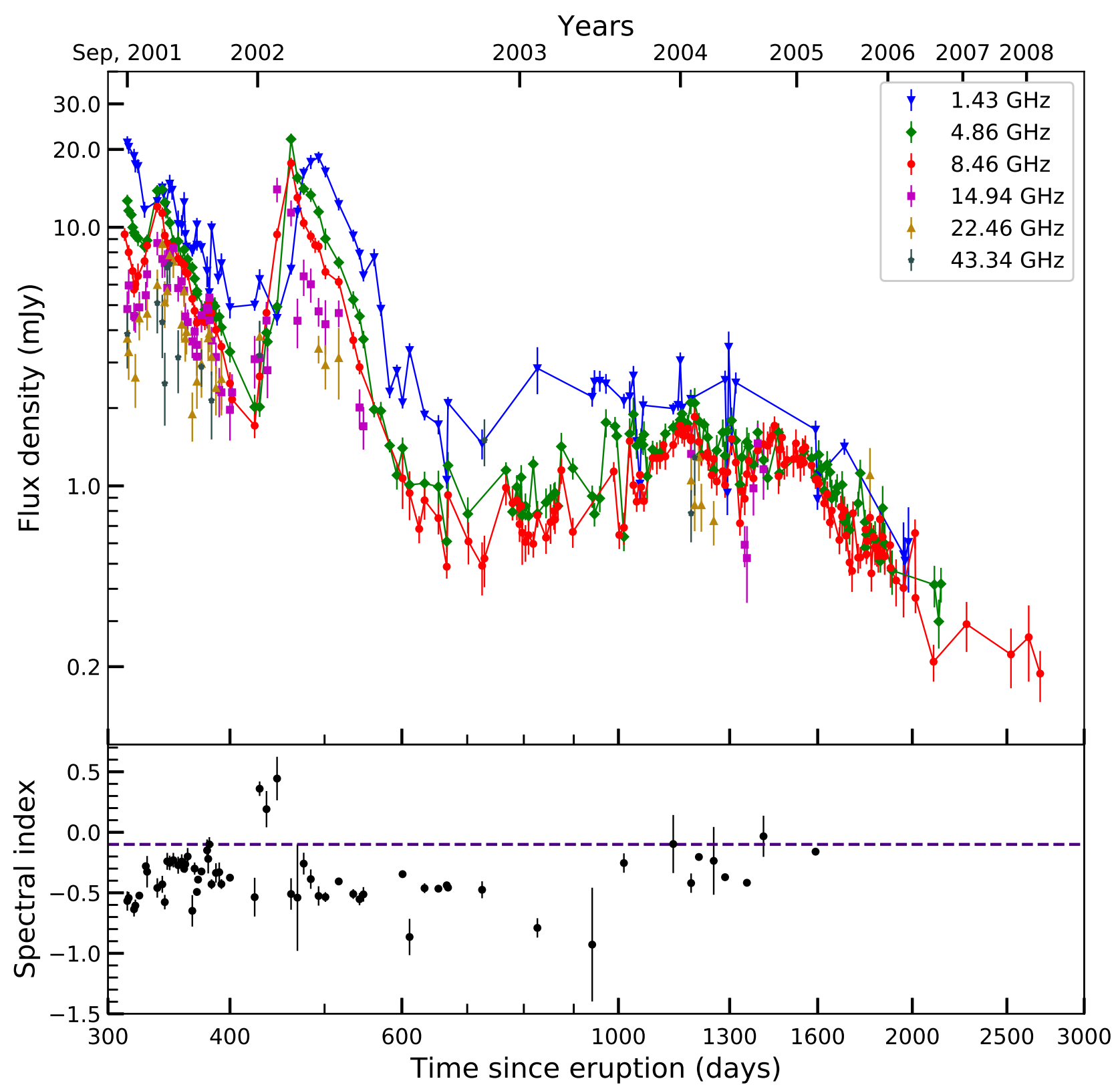

Figure 1. Top: Observed flux densities of V445 Pup spanning from day 300 to day 2700 after the nova eruption. We take 2000 November 02 as the date of the eruption $\left(t_{0}\right)$. Bottom: spectral indices obtained by fitting a single power-law to the data. The dashed line in the lower panel represents $\alpha=-0.1$, the theoretically expected index of optically thin free-free emission.

Table 4. High-Resolution A-configuration observations of V445 Pup.

\begin{tabular}{|c|c|c|c|c|}
\hline $\begin{array}{l}\text { Observation } \\
\text { Date Range }\end{array}$ & $\begin{array}{c}t-t_{0} \\
\text { (Days) }\end{array}$ & Description & $\begin{array}{c}\text { RA (J2000.0) } \\
(07 \mathrm{~h} 37 \mathrm{~m} 56 . \mathrm{XXXs})\end{array}$ & $\begin{array}{c}\text { Dec (J2000.0) } \\
\left(-25^{\circ} 56^{\prime} 58^{\prime \prime} . \mathrm{XX}\right)\end{array}$ \\
\hline 2001 Jan $18-$ Jan 30 & $77-89$ & Marginally detected & - & - \\
\hline 2002 Feb 7-May 15 & $462-559$ & Unresolved, FWHM $<0.1^{\prime \prime}$ & $.885 \pm .001$ & $.86 \pm .01$ \\
\hline 2003 May 17-Sep 19 & $926-1051$ & Marginally resolved, $0.3^{\prime \prime} \times<0.1^{\prime \prime}$ & $.880 \pm .001$ & $.85 \pm .02$ \\
\hline \multirow[t]{2}{*}{2004 Sep 23-2005 Jan 6} & $1408-1526$ & Two components separated by $0.2^{\prime \prime}$ & NE: $.890 \pm .002$ & $.80 \pm .03$ \\
\hline & & & SW: $.875 \pm .002$ & $.88 \pm .03$ \\
\hline 2006 Feb 9-May 10 & $1925-2015$ & One component, $0.3^{\prime \prime} \times<0.2^{\prime \prime}$ & $.883 \pm .002$ & $.85 \pm .04$ \\
\hline 2007 Jul 15-Jul 28 & $2446-2459$ & Marginally detected & - & - \\
\hline
\end{tabular}




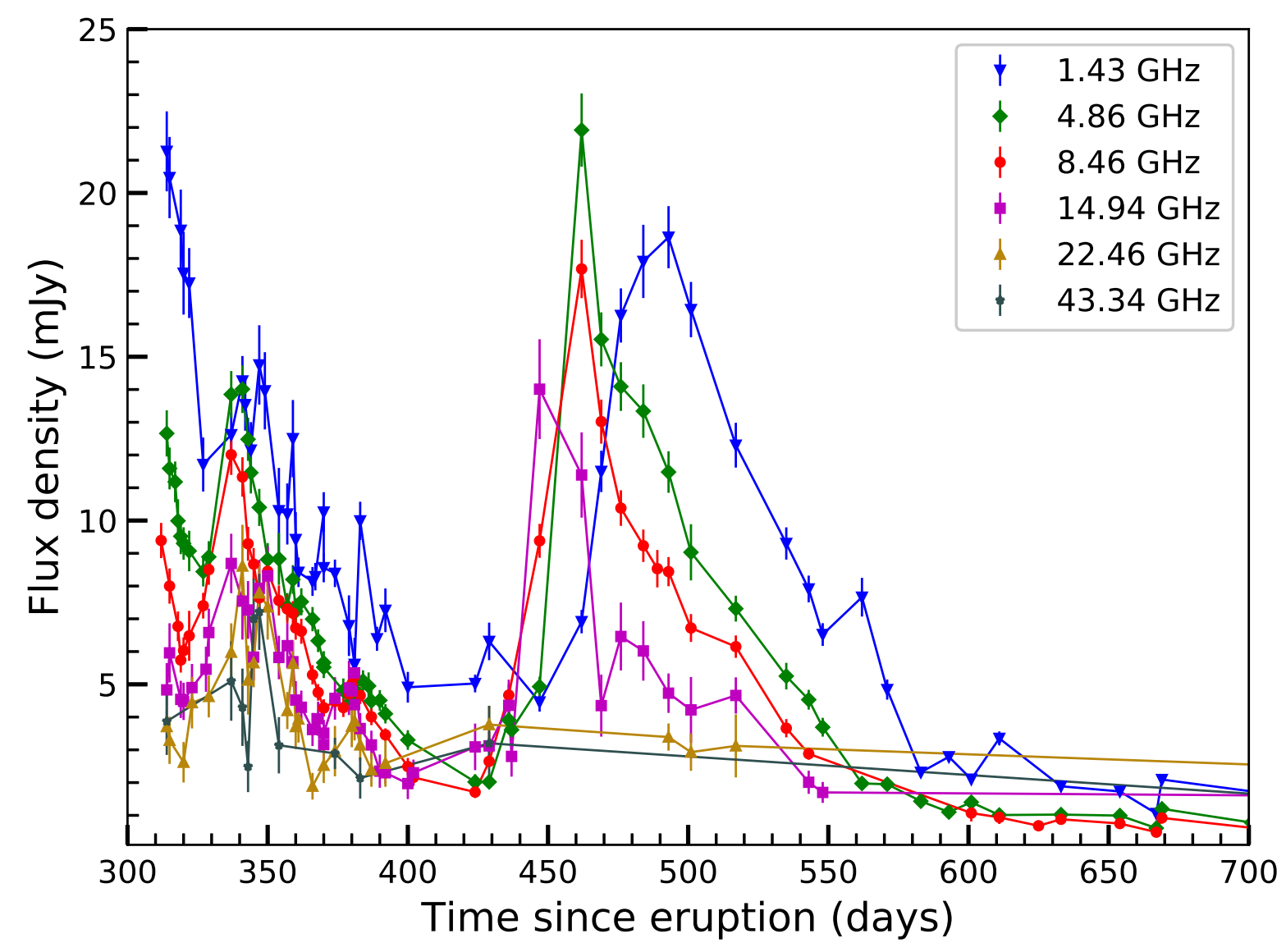

Figure 2. Observed flux densities of V445 Pup spanning from day 300 to day 700 after the nova eruption. Data are the same as those plotted in Figure 1, but zoomed in to show detail of the radio flares indicated with vertical dashed lines.

NAOS/CONICA adaptive optics system of the VLT. To make the near-IR K-band image, we downloaded several Kband exposures from the European Southern Observatory (ESO) Science Archive Facility (request \#543179), stacked them to remove cosmic rays and artifacts, and applied a world coordinate system (WCS) using six stars in the image with Gaia Data Release 2 (DR2) positions (estimated error on our WCS is $0.02^{\prime \prime}$ ). The near-IR K-band emission is a composite of emission lines from warm gas (e.g., He I) and warm dust continuum emission (Woudt et al. 2009).

From Figure 4, it is clear that the radio emission is emanating from the same bipolar regions of the V445 Pup ejecta that are dominating the near-IR emission. The two images were obtained around the same time (day $\sim 1445$ at $8.4 \mathrm{GHz}$, and day 1605 at near-IR K-band), and to first order, the two radio components are aligned with the peaks of the near-IR emission. Woudt et al. (2009) show that the thermal ejecta expand with a range of velocities in the polar (NE-SW) direction, while expansion in the equatorial plane is confined by a dense dust disc. It is the ejecta nearest to the dust disc that is densest and brightest in the near-IR - and also in radio emission.

We estimate the velocity at which the radio components are traveling apart from one another, assuming they were expelled on $t_{0}=51850$ MJD and V445 Pup is at a distance of $8.2 \mathrm{kpc}$. In convenient units, where $V$ is the expansion velocity in $\mathrm{km} \mathrm{s}^{-1}$ (assumed to be symmetric from a central location), $d$ is in kpc, $t$ is in units of 100 days, and $\theta$ is the angular separation in arcsec:

$V=8,660 \mathrm{~km} \mathrm{~s}^{-1}\left(\frac{d}{\mathrm{kpc}}\right)\left(\frac{\theta}{\operatorname{arcsec}}\right)\left(\frac{100 \text { days }}{t}\right)$

The component separation of $0.22^{\prime \prime}$ on day 1445 implies that the blobs were expanding at $1080 \mathrm{~km} \mathrm{~s}^{-1}$. If we estimate that both components have FWHM of $0.12^{\prime \prime}$, then the outer portions of the blobs would be separated by $0.34^{\prime \prime}$, implying expansion velocities of $1670 \mathrm{~km} \mathrm{~s}^{-1}$. Given these expansion velocities, it is not surprising that we do not observe significant motion during the 2004 A configuration; between 1408 and 1526 days after eruption, we would only expect the two components to move by $0.02-0.03^{\prime \prime}$ - substantially less than our observational errors (Table 4). The velocities of $1000-2000 \mathrm{~km} \mathrm{~s}^{-1}$ implied by the radio imaging are faster than the velocities of the $\mathrm{P}$ Cygni absorption troughs observed in optical spectroscopy a few months after eruption (Iijima \& Nakanishi 2008), but are slower than the fastest expanding ejecta imaged in the near-IR by Woudt et al. (2009).

We note that the high-velocity infrared "knots" pointed out at the extremities of the bipolar V445 Pup nebula by 


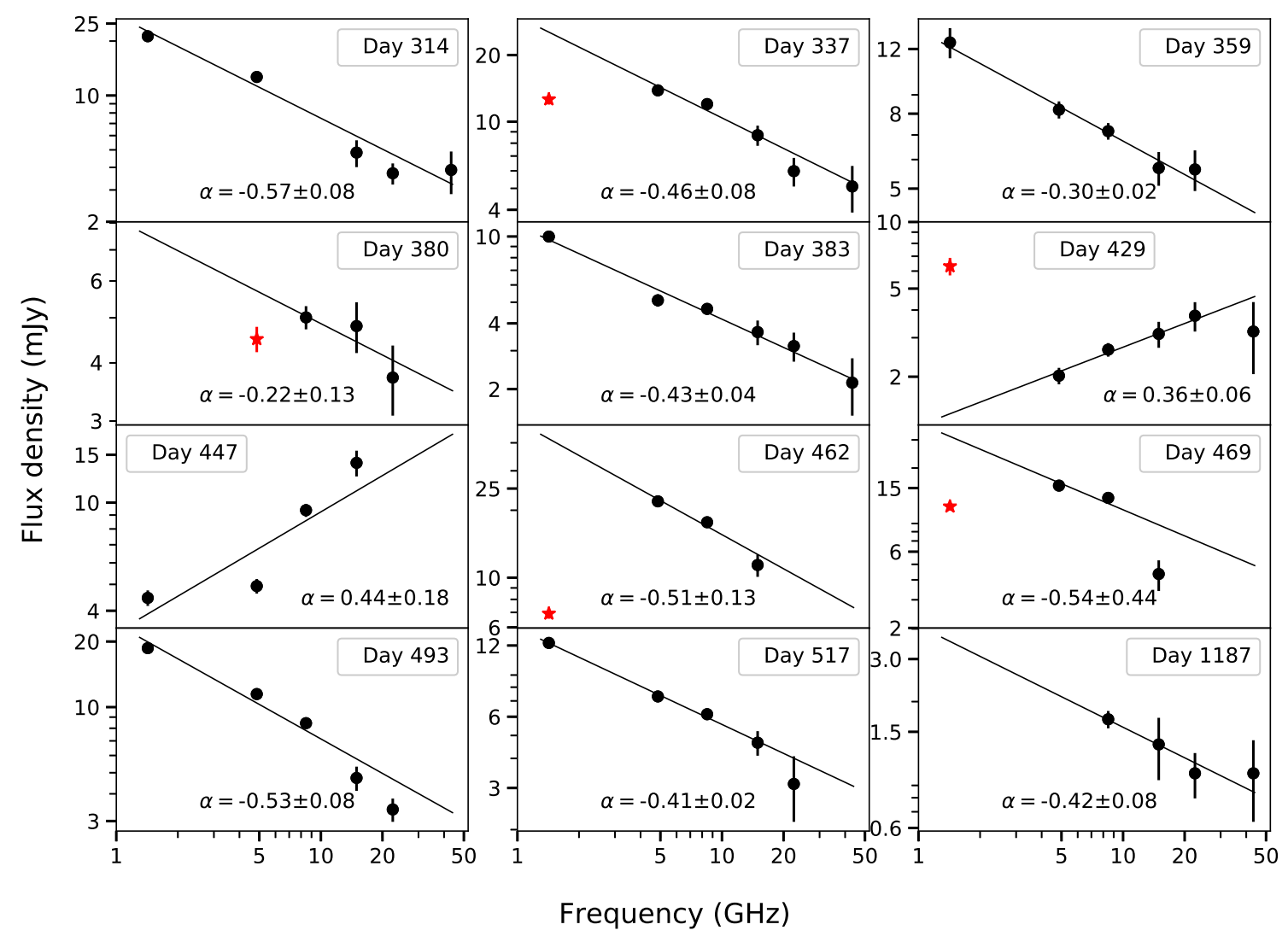

Figure 3. Selected VLA radio spectra of of V445 Pup, with linear fits overplotted. The time after eruption (since 2000 November 2) and the value of the spectral index is given for each spectrum. The data points marked with red stars represent flux densities in L-band (1.4 $\mathrm{GHz})$ or C-band $(4.9 \mathrm{GHz})$ which are relatively flat and thus not included in the fit.

Woudt et al. $(2009)^{1}$, seen to expand at a remarkable 8,450 $\mathrm{km} \mathrm{s}^{-1}$, would have been easily resolved in our radio images if they were radio bright. The high-velocity knots are not detected in our 2004 A configuration radio image - a clear demonstration that they are not the source of the bulk of the radio emission $\sim 1500$ days after eruption. Woudt et al. (2009) estimate that the high-velocity knots were ejected 345 days after $t_{0}$ (a few months before the 2002 A configuration imaging campaign). If the high-velocity knots had been radio-emitting during our 2003 A configuration campaign, they would have been separated by $\sim 0.7-0.8^{\prime \prime}$, easily resolvable with our high-resolution radio images. We therefore conclude that it is not the fastest-expanding material in V445 Pup that is dominating the radio emission at any time. Instead, our radio images imply that it is the densest material that is the site of the radio synchrotron emission.

In the 2006 A configuration (day 1925-2015), V445 Pup has substantially faded, and its morphology has reverted to

1 They are not visible in the K-band image presented in Figure 4; this may be because we did not stack all the exposures from 2005 Mar 26, and so our image is shallower than the one published by (Woudt et al. 2009). We also did not deconvolve the K-band image as done by Woudt et al. a similar structure as in 2003 (as best we can tell, given the relatively low $\mathrm{S} / \mathrm{N}$ ). We averaged in the $u v$-plane all 8.4 $\mathrm{GHz}$ data obtained in this A configuration, and found the data can be well-described by a single elliptical Gaussian with a major axis of $0.32^{\prime \prime} \pm 0.08^{\prime \prime}$, unresolved minor axis $\left(\lesssim 0.2^{\prime \prime}\right)$, and position angle of $68^{\circ} \pm 42^{\circ}$ (degrees east from north). The center of this emission region is consistent with the position of the 2003 emission; it is located between the 2004 components.

By the time of the 2007 A configuration, V445 Pup had faded too much for its morphology to be constrained by our VLA images.

\section{DISCUSSION}

\subsection{Radio Emission from V445 Pup is Synchrotron-Dominated}

Historically, radio emission from hydrogen-rich classical novae was thought to be dominated by thermal free-free radiation from expanding ionized ejecta (Seaquist \& Palimaka 1977; Hjellming et al. 1979; Seaquist \& Bode 2008). At early times, while the radio luminosity is increasing, the radioemitting region is optically thick. As the ejecta expand and 


\subsection{GHz (2004 Oct-Nov)}

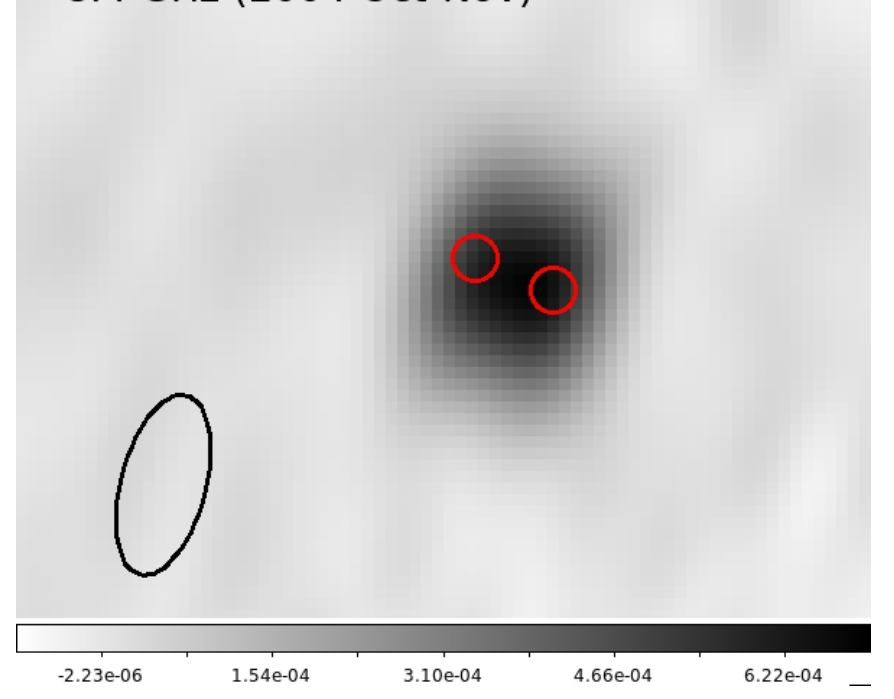

IR K band (2005 Mar)

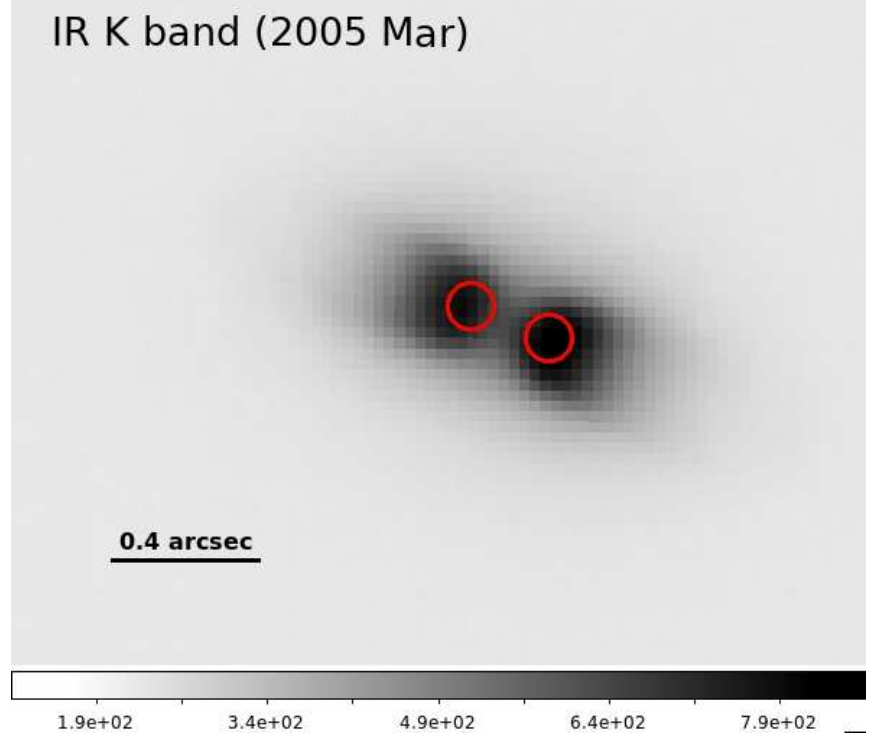

Figure 4. High-resolution images of V445 Pup observed at radio (left) and near-infrared (right) wavelengths. The red circles are the positions of the two Gaussian components fit to the 2004 Oct-Nov A configuration data in the $u v$ plane, and have diameters of $0.12^{\prime \prime}$. The left panel shows our $8.4 \mathrm{GHz}$ image representing the average of data obtained between 2004 Sep 23 and 2004 Nov 29 (days 1402-1488). The ellipse in the bottom left corner shows the FWHM extent of the synthesized beam $\left(0.48^{\prime \prime} \times 0.23^{\prime \prime}\right.$, PA $\left.=165^{\circ}\right)$. The right panel shows a near-IR K-band image obtained on 2005 Mar 26 (day 1605), with the same positions of the radio counterparts marked as red circles. In these images, north is up and east is to the left; and the fields-of-view are matched to be $2.4^{\prime \prime} \times 1.7^{\prime \prime}$. The grey scale is linear in both cases.

drop in density, the radio light curve peaks and turns over, as the radio photosphere recedes through the ejecta and the radio emission transitions to an optically thin state. During the optically thick phase of a thermal-dominated radio light curve, the spectral index $\alpha$ is expected to be equal to 2 , as for blackbody emission in the Rayleigh-Jeans long-wavelength limit. When the emitting region is optically thin, the spectral index is flat $(\alpha=-0.1)$, as expected for bremsstrahlung emission (e.g. $\S 6.2$ in Pacholczyk 1970). The radio light curve rise, peak, and decay occur on timescales of months to years - much slower than the evolution of optical light curves of novae.

The other possible source of nova radio emission is synchrotron radiation. The spectral index for optically thin synchrotron emission is set by the energy spectrum of relativistic electrons; if the number of relativistic electrons per unit energy is $N(E) \propto E^{-p}$, then the spectral index is $\alpha=(1-p) / 2$. For classic diffusive shock acceleration of relativistic particles, $p=2-2.5$, and so $\alpha=-0.5$ to -0.75 (Bell 1978; Blandford \& Ostriker 1978). Indeed, $\alpha \approx-0.7$ is commonly observed in synchrotron-emitting SNe and SN remnants. (Chevalier 1982; Weiler et al. 2002). However, several novae that are strong candidates for synchrotron emitters have unusually shallow spectral indices of $\alpha \approx-0.1$ to -0.5 (Taylor et al. 1987; Eyres et al. 2009; Weston et al. 2016a; Finzell et al. 2018). This could imply shallower energy spectra for relativistic electrons in novae (compared to e.g., $\mathrm{SNe}$ ), or that the synchrotron emitting region is inhomogeneous in terms of particle density and magnetic field strength (Vlasov et al. 2016). Optical depth effects could also flatten the spectrum; in the jets of active galactic nuclei, partially self-absorbed synchrotron emission routinely produces flat or inverted spectra of the "radio core", while the more extended transparent regions of the jet display the usual steep spectrum with $\alpha=-0.7$ (Eckart et al. 1986; Blandford et al. 2019).

The radio spectra of V445 Pup from $\sim 1-4.5$ years after optical discovery generally show higher flux densities at lower frequencies (Figure 3; with the exception of a few epochs which appear optically thick because of their inverted spectra). Spectral indices of V445 Pup are in the range $\alpha \approx 0$ to -1 (Figure 1). In the first three years of V445 Pup's evolution, its radio spectral index hovers around $\alpha=-0.5$ : slightly shallower than that observed for $\mathrm{SNe}$, but well within expectations of synchrotron emission (especially when taking into account the relatively shallow spectral indices observed for other synchrotron-emitting novae). After day $\sim 1000$, the spectral index flattens to $\alpha \approx-0.2$.

To further constrain the radio emission mechanism, we determine the brightness temperature $\left(T_{b}\right.$; a parameterization of surface brightness). Nova ejecta typically have temperatures $\sim 10^{4} \mathrm{~K}$, as a direct result of the photoionization of the ejecta by the central hot white dwarf (Cunningham et al. 2015). We therefore expect the brightness temperature to be $\sim 10^{4} \mathrm{~K}$ for a nova in the optically-thick thermal phase of its radio evolution. As thermal radio emission transitions to optically thin, the brightness temperature is expected to drop well below $10^{4} \mathrm{~K}$ (Weston et al. 2016a; Finzell et al. 2018). Synchrotron emission, on the other hand, can reach much higher brightness temperatures, up to $\sim 10^{11} \mathrm{~K}$ (Readhead 1994). Therefore, if the nova's radio brightness temperature is substantially in excess of $10^{4} \mathrm{~K}$, this is a promising indication that it is emitting non-thermal radiation.

To estimate the brightness temperature, we must con- 


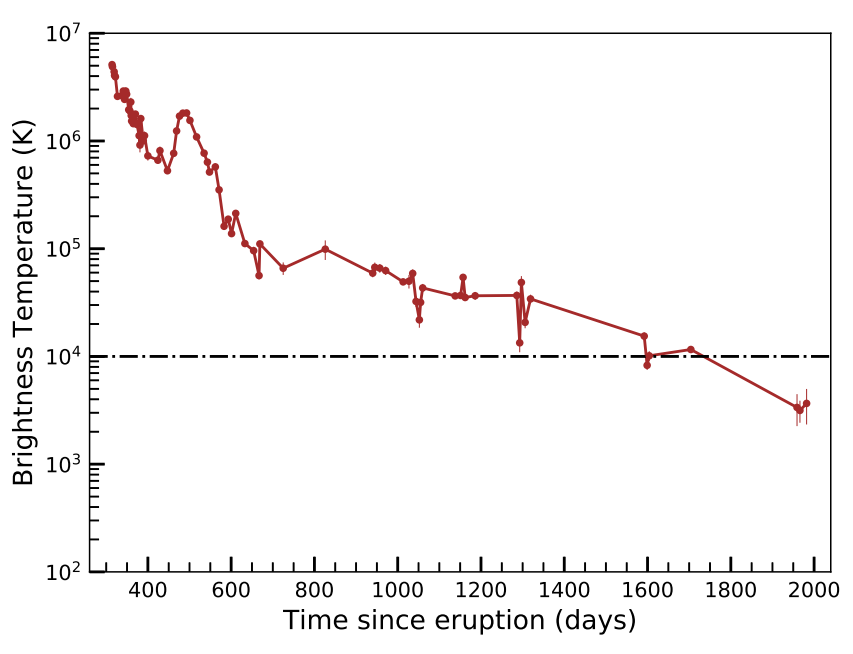

Figure 5. Surface brightness temperature for V445 Pup between 2001 September and 2006 April.

strain the angular diameter of the object $(\theta)$ and measure its flux density $\left(S_{V}\right)$. We can then calculate the brightness temperature using the formula:

$\frac{T_{b}}{\mathrm{~K}}=1.36 \times\left(\frac{\lambda}{\mathrm{cm}}\right)^{2} \times\left(\frac{S_{v}}{\mathrm{mJy}}\right) \times\left(\frac{\theta_{a} \theta_{b}}{\operatorname{arcsec}^{2}}\right)^{-1}$

where $\lambda$ is the observing wavelength, $\theta_{a}$ is the major axis diameter, and $\theta_{b}$ is the minor axis diameter. We can estimate $\theta$ if we know the speed at which the nova ejecta are expanding $(V$; equation 1$)$ as a function of time $(t$; Seaquist \& Bode 2008). We take $t$ as the time since $t_{0}$ and estimate the expansion velocity $V=1600 \mathrm{~km} \mathrm{~s}^{-1}$ along the major axis and $V<800 \mathrm{~km} \mathrm{~s}^{-1}$ along the minor axis (inferred from radio imaging; §3.3). We use the observed flux densities at 1.43 $\mathrm{GHz}(21 \mathrm{~cm})$, which place the strongest constraints on the brightness temperature (compared to higher frequencies).

As seen in Figure 5, the brightness temperature of V445 Pup starts high, $\sim 10^{7} \mathrm{~K}$ on day $\sim 300$, implying that it is undeniably synchrotron emission. The brightness temperature declines with time, to $\sim 10^{5} \mathrm{~K}$ by day $\sim 600$, and finally to $\sim 10^{4} \mathrm{~K}$ by day $\sim 2,000$. Despite a relatively low (for synchrotron emission) brightness temperature of few $\times 10^{4} \mathrm{~K}$ after $\sim 1000$ days, we conclude that this emission is non-thermal, since the spectral index implies that the emission is optically-thin. If the emission were optically-thin thermal, its brightness temperature should be substantially lower than the $\sim 10^{4} \mathrm{~K}$ electron temperature. Therefore, we conclude that the radio light curve of V445 Pup is dominated by synchrotron emission over $\sim 7$ years of its evolution.

\subsection{What powers the synchrotron-emitting shocks in V445 Pup?}

The general consensus is that the source of synchrotron emission in novae is due to acceleration of particles to relativistic speeds in shocks. Depending on the nature of the companion star, these shocks can be either external with pre-existing circumbinary material or internal within the ejecta. Which is it for V445 Pup?

The clearest examples of synchrotron emission in hydrogen-rich classical novae come from embedded novae with red giant companions, where external shocks are produced in the interaction of the nova ejecta and the red giant wind. Examples of this type of synchrotron-emitting shocks are RS Oph (O'Brien et al. 2006; Rupen et al. 2008; Sokoloski et al. 2008; Eyres et al. 2009), V745 Sco (Kantharia et al. 2016), and V1535 Sco (Linford et al. 2017). In these cases, the radio light curve evolves relatively quickly (over $\sim$ weeks), and can be used to trace the radial density profile of the circumbinary material. A late-time thermal radio component is not observed in most cases, indicating that the ejecta are low mass (as expected for the relatively high accretion rates driven by red giant companions; e.g., Yaron et al. 2005).

Another way to produce synchrotron emission could be internal shocks within the ejecta. These type of shocks likely dominate in a white dwarf system whose companion is a main sequence star, and hence the binary system is surrounded by less dense circumbinary material. Such shocks form at the interface of two outflows moving at different speeds, following a nova eruption. For decades there has been evidence of internal shocks in hydrogen-rich classical novae, based on hard X-ray emission (O'Brien et al. 1994; Mukai \& Ishida 2001; Mukai et al. 2008), and more recently, $\mathrm{GeV} \gamma$-ray emission (Ackermann et al. 2014; Franckowiak et al. 2018; Martin et al. 2018). It has also recently been recognized that a significant fraction of these novae show evidence of non-thermal radio emission, manifesting as early bright radio flares. Novae showing evidence of synchrotron emission from internal shocks include QU Vul (Taylor et al. 1987), V959 Mon (Chomiuk et al. 2014), V1723 Aql (Weston et al. 2016a), V5589 Sgr (Weston et al. 2016b), and V1324 Sco (Finzell et al. 2018). These flares rise rapidly with time ( $\sim$ days-weeks), in contrast to expectations for expanding thermal ejecta (rise time $\approx$ months), and have brightness temperatures $\gtrsim 10^{5}-10^{6} \mathrm{~K}$. In most cases, a second radio maximum becomes visible at late times which is well-described as expanding thermally emitting ejecta.

Is V445 Pup's radio synchrotron emission driven by external or internal shocks? We know little about the companion star due to dust obscuration (Ashok \& Banerjee 2003; Woudt et al. 2009), so it is difficult to predict the properties of the circumbinary material surrounding V445 Pup. We can rule out external shocks with relatively sphericallydistributed circumbinary material, based on our radio imaging (§3.3). If the pre-existing material was isotropically distributed, we would expect the synchrotron emission to be brightest at the fastest shocks (see Equation 3), and so to be easily resolvable (as, e.g., the near-IR high-velocity knots; Woudt et al. 2009). Instead, the radio emission is confined to much nearer the binary.

Based on radio imaging of V959 Mon, Chomiuk et al. (2014) hypothesize that at the beginning of a nova eruption, a slow outflow concentrated in the equatorial plane of the binary system is generated. A more isotropic fast wind then follows, primarily escaping in the polar directions. The collision between the two distinct flows results in shocks and particle acceleration. Based on near-infrared and radio imaging in the years following the 2000 eruption, V445 Pup exhibits a similar bipolar outflow and an equatorial disc (Woudt et al. 2009). Our radio imaging reveals that the synchrotron emission is concentrated near the equatorial 
plane, even $3-5$ years after explosion ( $\$ 3.3$, Figure 4 ). We also know that the equatorial disc maintains its structure for years following the eruption (Woudt et al. 2009). Therefore, interactions between a polar flow and equatorial disc, as proposed for V959 Mon, are the most likely source of the synchrotron emission.

The origin of the equatorial disc, however, is unclear. It may have pre-dated the nova eruption, as mass lost in a previous nova eruption, or from the binary during quiescence (perhaps from the outer Lagrange points during mass transfer; e.g., Pejcha et al. 2016a). Indeed, from pre-eruption photometry, Woudt et al. (2009) deduced substantial circumbinary dust around V445 Pup in quiescence. The very strong IR signatures of dust early in the eruption led Lynch et al. (2001) to surmise that the dust may have pre-dated the eruption, a conclusion also reached by Shimamoto et al. (2017) when they measured very large dust masses $\left(\sim 5 \times 10^{-4}\right.$ $\mathrm{M}_{\odot}$ ) around V445 Pup in 2006. On the other hand, in at least the case of V959 Mon, an equatorial disc-like structure was ejected during the nova eruption itself (Chomiuk et al. 2014; Linford et al. 2015). It is hypothesized that the equatorial structure was produced by the puffed-up nova envelope, marginally bound to the binary shortly after thermonuclear runaway (Chomiuk et al. 2014). As the binary orbited inside the envelope, it transferred energy to help the envelope expand, preferentially in the equatorial direction (i.e., Pejcha et al. 2016b). It is worth noting that, unlike in V959 Mon, the equatorial disc is not observed to expand or diffuse during the eruption of V445 Pup; it remains present in imaging until at least 2015, and significant dust obscuration persists around the binary up until the present day (Woudt et al. 2021, in prep). This relative constancy of the equatorial disc over $\sim$ two decades may suggest a pre-eruption origin.

\subsection{Understanding the synchrotron-dominated light curve of V445 Pup}

The radio synchrotron emission from V445 Pup persists for an unprecedentedly long time ( $\sim 7$ years; Figure 1$)$. Synchrotron emission in other novae lasts for just a few weeks-months (Eyres et al. 2009; Weston et al. 2016a,b; Kantharia et al. 2016; Linford et al. 2017). One relatively simple way to explain the long synchrotron duration of V445 Pup would be if a wind was launched from the binary and continued blowing for years after eruption, while the equatorial disc retained its structure over this time. Then ongoing interactions between the wind and the disc would continue to power synchrotron luminosity. The standard mechanism for driving prolonged winds in novae is radiation pressure from an $\sim$ Eddington luminosity white dwarf, powered by nuclear burning on the white dwarf's surface (Kato \& Hachisu 1994). While $\sim 7$ years is relatively long for this sustained burning phase, it is not unprecedented amongst hydrogen-rich classical novae (Henze et al. 2014). The larger envelope masses in helium novae lead to expectations of longer durations for the sustained burning phase in systems like V445 Pup ( few yr - 10,000 yr; Kato \& Hachisu 2004). Therefore, a wind prolonged over $\sim 7$ years seems reasonable. Unfortunately, we are not able to directly test if a wind in V445 Pup was powered by the nuclearburning white dwarf, as no X-ray observations of the erup- tion are available, and the dusty equatorial disc would likely have absorbed the white dwarf supersoft X-ray emission.

To explain the synchrotron emission from V445 Pup, we consider a cartoon model where the white dwarf wind impacts upon the equatorial disc (we assume the disc is not expanding, as it has appeared largely unchanged in imaging over $\sim 15$ years post eruption; Woudt et al. 2009, Woudt et al. 2021, in prep.). We approximate that the wind expands at $1600 \mathrm{~km} \mathrm{~s}^{-1}$, based on our radio imaging (§3.3), noting that this is the measured velocity in the polar (NE-SW) direction. We take a scenario where a wind of this velocity emanates from the white dwarf isotropically, but is decelerated in the equatorial direction by the disc, creating shocks, accelerating particles, and producing the observed synchrotron emission. We use a simple prescription for synchrotron luminosity, as described in Appendix A and largely taken from Chevalier $(1982,1998)$. We assume a fraction of the postshock energy density is transferred to energy density of the amplified magnetic field $\left(U_{B}=\varepsilon_{B} \rho_{\text {disc }} v_{\mathrm{w}}^{2}\right)$ and of relativistic electrons $\left(U_{e}=\varepsilon_{e} \rho_{\mathrm{disc}} v_{\mathrm{w}}^{2}\right)$. Here $\rho_{\mathrm{disc}}$ is the density of the pre-shock material and $v_{\mathrm{w}}$ is the velocity of the wind, $\varepsilon_{B}$ and $\varepsilon_{e}$ represent the fraction of the post-shock energy due to amplified magnetic fields and the relativistic electrons, respectively. We note that this is likely an oversimplification if the wind significantly decelerates in the equatorial direction, implying that a reverse shock contributes to the shock luminosity (Metzger et al. 2014). A thorough treatment of V445 Pup's shocks and resultant synchrotron luminosity requires a multi-dimensional hydrodynamics simulation, so our goal here is only to draw a simple cartoon. When the synchrotron emission is optically thin, its luminosity $\left(L_{V}\right)$ is proportional to the synchrotron-emitting volume $V_{\text {synch }} \times U_{e} \times U_{B}^{(p+1) / 4}$. For V445 Pup, the index of the relativistic electron energy spectrum is measured to be $p \approx 2$ (§4.1); therefore

$$
L_{v} \propto V_{\text {synch }} \varepsilon_{e} \varepsilon_{B}^{3 / 4} \rho_{\text {disc }}^{7 / 4} v_{\mathrm{w}}^{7 / 2}
$$

As described in Appendix A, the synchrotron luminosity of V445 Pup can be explained if $\rho_{\text {disc }} \approx 10^{4} \mathrm{~cm}^{-3}$.

Higher synchrotron luminosities imply faster shocks and/or higher densities for interaction. Therefore, the variations in synchrotron luminosity observed in V445 Pup's light curve imply variations in the wind velocity or density of the equatorial disc - or in the optical depth. Synchrotrondominated light curves of radio transients often show an optically-thick rise, where the flux increases as $\tau$ drops, and an optically-thin decline, usually interpreted as a decline in the density of material being shocked and/or a decline in the shock velocity (e.g., Weiler et al. 2002). V445 Pup is unusual in showing multiple peaks in its light curve (although see the embedded nova V1535 Sco for a similar albeit less dramatic case; Linford et al. 2017).

These flares are accompanied by changes in the optical depth. While most of the time the synchrotron emission appears optically thin (flux density monotonically rising toward lower frequency or $\alpha<0$ ), on the rise to radio peaks, the radio spectrum flips to be brighter at higher frequencies (Figures 1 and 3). Between days 429 and 447 (on the rise to the brightest flare observed, peaking on day $\sim 460$ ), the flux density increases with frequency all the way up to $22.5 \mathrm{GHz}$, implying $\alpha=0.2$ to 0.4 . On days 337-341 (rising to a slightly fainter peak on day $\sim 340$ ), the $1.4 \mathrm{GHz}$ 
point falls substantially below the power law fit to higherfrequencies, implying that absorption is present but milder than on day 429. In both cases, the radio spectrum returns to optically thin at the peak of the radio flare, and thereafter. The observed cyclic change in the radio spectrum of V445 Pup - starting with optically thin emission, then becoming optically-thick, then thin, and then thick and thin again - is unusual and challenging to explain (although again, similar sudden, cyclic changes of $\alpha$ are seen in V1535 Sco; Linford et al. 2017). With a few rough calculations, we can exclude the possibility that the optical depth is due to synchrotron self-absorption (Appendix B). The optical depth is therefore due to free-free absorption, and changes in opacity mean that the ionization state of the absorbing material must be changing. The spectrum never reaches the canonical $\alpha=2$ for free-free absorption, implying that the emission is only partially optically thick-perhaps because the $\tau \approx 1$ photospheres shrink with frequency as expected in a stellar wind (Panagia \& Felli 1975; Wright \& Barlow 1975 ) or because the covering factor of the absorbing screen is substantially smaller than unity (e.g., Diaz et al. 2018).

So, what might explain the observed radio flares, and attending changes in free-free opacity? One possibility is that the white dwarf wind is sweeping through the inner parts of the equatorial disc, and encountering density enhancements within it. This would imply that there are at least four regions of enhanced density in the disc, to explain the flares on day $<300$ (we only captured its decline), day 340 , day 460 , and day $\sim 1300$ (Figure 1). Simulations of equatorial discs produced by mass loss from the outer Lagrangian points of a mass-transferring binary often find structured discs with spiral-like density enhancements (Pejcha et al. $2016 \mathrm{a}, \mathrm{b})$. The flux of V445 Pup rose by a factor of $\sim 10$ (from $2 \mathrm{mJy}$ to $22 \mathrm{mJy}$ at $4.9 \mathrm{GHz}$ ) between day 430 and 460. According to Equation 3, this would imply an increase in density of a factor of $\sim 4$ to explain this flare.

This interpretation is supported by the rapid declines of the radio flares, which we parameterize as $S_{v} \propto t^{\beta}$; the value of $\beta$ can give hints on the source of the synchrotron emission. Harris et al. (2016) performed hydrodynamic simulations of a shock interacting with "shells" of enhanced density. During impact with a shell, the energy densities in relativistic electrons and magnetic fields increase dramatically, and therefore the radio light curve will peak. However, as the shock propagates to the outer side of the shell, there is no more material to sweep up, particle acceleration ceases, and the energy densities in relativistic electrons and magnetic fields rapidly drop as expected for adiabatic expansion. At this point the radio light curves decline rapidly as $L_{v} \propto t^{-11.5}$ to -9 based on their simulations and analytical relations. Considering the declines from the radio flares on days 337 and 462 (see Figure 2), $\beta$ is in the range $-10 \lesssim \beta \lesssim-9$, consistent with the shell-interaction simulations.

The other possibility is that the velocity of the wind is variable with time, and synchrotron flares are produced when it is faster. To produce a factor of ten increase in synchrotron luminosity requires a factor of $\sim 2$ increase in the wind velocity, according to Equation 3. Other nova eruptions have been observed to host multiple outflows with a range of velocities, seen in optical spectra as new absorption features and broadening emission lines (e.g., Jack et al.
2017; Aydi et al. 2019). Aydi et al. (2020) observed rapid changes in $\gamma$-ray flux and shock luminosity over the first $\sim 40$ days of V906 Car's 2018 nova eruption, and attributed this to changes in outflow velocity with support from optical spectroscopy. However, the flares observed in V906 Car, and indeed most hydrogen-rich novae, occur over substantially shorter periods than the $\sim$ few years covered by our V445 Pup radio light curve. Perhaps helium nova eruptions evolve more slowly due to their likely larger envelope masses. However, this is difficult to test, as there was essentially no spectroscopic monitoring of V445 Pup during the time window 1-3 years after explosion, due to very heavy dust obscuration (Ashok \& Banerjee 2003; Lynch et al. 2004; Iijima \& Nakanishi 2008), and so it is difficult to constrain if or how the wind velocity changed during this time.

In either case - whether it is variations in density or velocity that produce the radio flares - the likely cause of the changes in optical depth is changes in shock luminosity. If much of this shock luminosity is radiated in the ultraviolet band, it can ionize portions of the equatorial disc ahead of it. The higher the shock luminosity, the more ionized material there will be, and the stronger the free-free absorption. The free-free absorption later dissipates, either because this material recombines or because it is swept up by the shock. We prefer the latter explanation, because if the disc material has a density $\sim 10^{4} \mathrm{~cm}^{-3}$ (Appendix A), the recombination time should be of order a century (Ferland 2003): much longer than the $\sim 200$ day duration of the radio flare.

\subsection{Is V445 Pup a SN Ia progenitor system?}

As the only helium nova known, V445 Pup is often singled out as an intriguing candidate for a progenitor system to SNe Ia (e.g., Li et al. 2011; McCully et al. 2014; Kelly et al. 2014). The progenitors of SNe Ia have been constrained with radio continuum observations, where the synchrotronemitting blast wave formalism of Chevalier and collaborators (i.e., Appendix A) is used to place limits on the density and distribution of circumstellar material near the SN site (e.g., Panagia et al. 2006; Chomiuk et al. 2016; Lundqvist et al. 2020). Tested configurations of circumstellar material are usually over-simplistic, with smooth density profiles and spherical symmetry. Therefore interpretations of radio limits for SNe Ia have substantial associated uncertainty.

The fact that V445 Pup's radio light curve is synchrotron dominated and powered by interaction with an equatorial disc that likely pre-dated the nova eruption presents a unique opportunity to predict what a radio light curve of a realistic SN Ia might look like. We had originally hoped that we could simply "scale up" from nova ejecta energetics to SN ejecta energetics to predict how a SN exploding in a V445 Pup-like progenitor system would appear at radio wavelengths. However, there are two problems with this strategy. First, the synchrotron emission in V445 Pup was likely produced by a prolonged wind that continued to blow and interact with the disc for years - very different from the impulsive yet homologous explosions of SNe. Second, while the equatorial disc apparently withstood the nova eruption of V445 Pup, it is not clear if it would be destroyed by SN Ia ejecta - and on what timescale.

In the future, hydrodynamic simulations should model 
the V445 Pup wind/disc system and work to constrain the mass loss rate of the white dwarf wind and the density profile of the equatorial disc, using our radio light curve as a critical constraint on the dynamics. This would inform the ejecta mass and energetics of the only helium nova known, and also recreate the circumstellar environment of one of the most promising SN Ia progenitor systems. Once the mass, extent, and morphology of the equatorial disc are better constrained, another hydrodynamic simulation could model the interaction of a SN Ia-like explosion with the disc, and make real predictions for observable signatures at radio, optical, and X-ray wavelengths that might indicate a V445 Pup-like progenitor (see Booth et al. 2016 for a similar strategy implemented on another SN Ia progenitor candidate, RS Oph).

\section{CONCLUSIONS}

The helium nova V445 Pup is observed at radio frequencies for years following its eruption in late 2000 (Figure 1). Steep spectral indices and high brightness temperatures imply that the radio light curve is powered by synchrotron emission through 2008; we see no evidence of thermal emission from an expanding ionized nova remnant, as seen in many other novae at radio wavelengths. The radio light curve is characterised by at least four re-brightening events, which sometimes are subject to free-free absorption on the rise but revert back to optically-thin synchrotron emission as they fade. Spatially-resolved radio images show that the synchrotron emission is more compact than the thermal ejecta and is confined near the equatorial disc imaged at near IR wavelengths (Figure 4).

We hypothesise that a wind from the white dwarf interacts with the equatorial disc, giving rise to shocks, particle acceleration, and synchrotron emission. This model is very similar to the scenario proposed to explain radio synchrotron and $\mathrm{GeV} \gamma$-ray emission in hydrogen-rich classical nova V959 Mon, also based on radio imaging (Chomiuk et al. 2014). However, the data presented here on V445 Pup present the clearest case to date of synchrotron emission being associated with a dense equatorial disc in a nova. We note that, based on its high synchrotron luminosity and detection of $\gamma$-rays in other novae (Ackermann et al. 2014; Franckowiak et al. 2018), V445 Pup was likely a source of substantial GeV $\gamma$-rays, but unfortunately its eruption occurred between the Compton Gamma-Ray Observatory and Fermi Gamma-Ray Space Telescope missions.

The duration of bright synchrotron emission from V445 Pup ( $~ 7$ years) is unprecedented amongst novae. In our model, this implies that the equatorial disc must have maintained its structure throughout these years (a hypothesis directly supported by near IR imaging of V445 Pup; Woudt et al. 2009). In addition, the wind from the white dwarf must have persisted over these years, which is not surprising if the wind is powered by radiation pressure from sustained burning of helium on the white dwarf's surface (Kato \& Hachisu 2004). The re-brightening events in the radio light curve could be produced by changes in wind velocity or density enhancements encountered in the disc, as it is swept up by the wind. Similar fluctuations in shock luminosity have been seen in GeV $\gamma$-rays - albeit over shorter timescales - in the 2018 nova V906 Car (Aydi et al. 2020).

\section{ACKNOWLEDGEMENTS}

The authors are grateful to Dr. Chelsea E. Harris and Dr. Ken J. Shen for useful discussions. They also thank Prof. Michael Rupen, Dr. Amy Mioduszewski and Dr. Vivek Dhawan for their work in obtaining the VLA observations presented here. The National Radio Astronomy Observatory is a facility of the National Science Foundation operated under cooperative agreement by Associated Universities, Inc.

This research was supported by the South African Radio Astronomy Observatory, which is a facility of the National Research Foundation (NRF), an agency of the Department of Science and Innovation. MMN and PAW kindly acknowledge financial support from the University of Cape Town and the NRF. V.A.R.M.R. acknowledges financial support from the Fundação para a Ciência e a Tecnologia (FCT) in the form of an exploratory project of reference IF/00498/2015/CP1302/CT0001, FCT and the Ministério da Ciência, Tecnologia e Ensino Superior (MCTES) through national funds and when applicable co-funded EU funds under the project UIDB/EEA/50008/2020, and supported by Enabling Green E-science for the Square Kilometre Array Research Infrastructure (ENGAGE-SKA), POCI-01-0145FEDER-022217, and PHOBOS, POCI-01-0145-FEDER029932, funded by Programa Operacional Competitividade e Internacionalização (COMPETE 2020) and FCT, Portugal. L.C. and K.V.S. acknowledge financial support of NSF awards NSF AST-1751874 \& AST-1907790, NASA grants Fermi/80NSSC18K1746 \& NuSTAR/80NSSC19K0522, and a Cottrell fellowship of the Research Corporation. J.S. acknowledges support from the Packard Foundation.

Software: CASA (McMullin et al. 2007), AIPS (Greisen 2003b), difmap (Shepherd 1997b), SciPy (Oliphant 2007), Numpy (van der Walt et al. 2011), matplotlib (Hunter 2007).

\section{DATA AVAILABILITY}

The data presented in this work are available through the VLA archive which can be accessed via https://archive.nrao.edu/archive/advquery.jsp.

\section{REFERENCES}

Ackermann M., et al., 2014, Science, 345, 554

Ashok N. M., Banerjee D. P. K., 2001, IAU Circ., 7559, 2

Ashok N. M., Banerjee D. P. K., 2003, A\&A, 409, 1007

Aydi E., et al., 2019, arXiv e-prints, p. arXiv:1903.09232

Aydi E., et al., 2020, Nature Astronomy,

Bell A. R., 1978, MNRAS, 182, 147

Bell A. R., 2004, MNRAS, 353, 550

Blandford R. D., Ostriker J. P., 1978, ApJ, 221, L29

Blandford R., Meier D., Readhead A., 2019, ARA\&A, 57, 467

Bode M. F., Evans A., 2008, Classical Novae

Booth R. A., Mohamed S., Podsiadlowski P., 2016, MNRAS, 457,822

Cendes Y., Drout M. R., Chomiuk L., Sarbadhicary S. K., 2020, ApJ, 894, 39

Chevalier R. A., 1982, ApJ, 259, 302

Chevalier R. A., 1998, ApJ, 499, 810

Chevalier R. A., Fransson C., 2006, ApJ, 651, 381 
Chomiuk L., et al., 2012, ApJ, 750, 164

Chomiuk L., et al., 2014, Nature, 514, 339

Chomiuk L., et al., 2016, ApJ, 821, 119

Cunningham T., Wolf W. M., Bildsten L., 2015, ApJ, 803, 76

Diaz M. P., Abraham Z., Ribeiro V. A. R. M., Beaklini P. P. B., Takeda L., 2018, MNRAS, 480, L54

Eckart A., Witzel A., Biermann P., Johnston K. J., Simon R., Schalinski C., Kuhr H., 1986, A\&A, 168, 17

Eyres S. P. S., et al., 2009, MNRAS, 395, 1533

Ferland G. J., 2003, ARA\&A, 41, 517

Finzell T., et al., 2018, ApJ, 852, 108

Franckowiak A., Jean P., Wood M., Cheung C. C., Buson S., 2018, A\&A, 609, A120

Greisen E. W., 2003a, AIPS, the VLA, and the VLBA. p. 109, doi:10.1007/0-306-48080-8_7

Greisen E. W., 2003b, AIPS, the VLA, and the VLBA. p. 109, doi:10.1007/0-306-48080-8_7

Harris C. E., Nugent P. E., Kasen D. N., 2016, ApJ, 823, 100

Henze M., et al., 2014, A\&A, 563, A2

Hillman Y., Prialnik D., Kovetz A., Shara M. M., 2016, ApJ, 819, 168

Hjellming R. M., Wade C. M., Vandenberg N. R., Newell R. T., 1979, AJ, 84, 1619

Hunter J. D., 2007, Computing in Science and Engineering, 9, 90

Iijima T., Nakanishi H., 2008, A\&A, 482, 865

Jack D., et al., 2017, Astronomische Nachrichten, 338, 91

Kantharia N. G., 2012, Bulletin of the Astronomical Society of India, 40, 311

Kantharia N. G., et al., 2016, MNRAS, 456, L49

Kato M., Hachisu I., 1994, ApJ, 437, 802

Kato M., Hachisu I., 2004, ApJ, 613, L129

Kato M., Saio H., Hachisu I., 1989, ApJ, 340, 509

Kato T., Kanatsu K., Takamizawa K., Takao A., Stubbings R., 2000, IAU Circ., 7552, 1

Kelly P. L., et al., 2014, ApJ, 790, 3

Kundu E., Lundqvist P., Pérez-Torres M. A., Herrero-Illana R., Alberdi A., 2017, ApJ, 842, 17

Li W., et al., 2011, Nature, 480, 348

Linford J. D., et al., 2015, ApJ, 805, 136

Linford J. D., et al., 2017, ApJ, 842, 73

Lundqvist P., et al., 2020, ApJ, 890, 159

Lynch D. K., Russell R. W., Sitko M. L., 2001, AJ, 122, 3313

Lynch D. K., Rudy R. J., Mazuk S., Venturini C. C., Puetter R. C., Perry R. B., 2004, AJ, 128, 2962

Martin P., Dubus G., Jean P., Tatischeff V., Dosne C., 2018, A\&A, 612, A38

McCully C., et al., 2014, Nature, 512, 54

McMullin J. P., Waters B., Schiebel D., Young W., Golap K., 2007, in Shaw R. A., Hill F., Bell D. J., eds, Astronomical Society of the Pacific Conference Series Vol. 376, Astronomical Data Analysis Software and Systems XVI. p. 127

Metzger B. D., Hascoët R., Vurm I., Beloborodov A. M., Chomiuk L., Sokoloski J. L., Nelson T., 2014, MNRAS, 442, 713

Moll R., Woosley S. E., 2013, ApJ, 774, 137

Mukai K., Ishida M., 2001, ApJ, 551, 1024

Mukai K., Orio M., Della Valle M., 2008, ApJ, 677, 1248

Nomoto K., 1982, ApJ, 257, 780

O'Brien T. J., Lloyd H. M., Bode M. F., 1994, MNRAS, 271, 155

O'Brien T. J., et al., 2006, Nature, 442, 279

Offringa A. R., van de Gronde J. J., Roerdink J. B. T. M., 2012, A\&A, 539, A95

Oliphant T. E., 2007, Computing in Science and Engineering, 9,10

Pacholczyk A. G., 1970, Radio astrophysics. Nonthermal processes in galactic and extragalactic sources

Panagia N., Felli M., 1975, A\&A, 39, 1

Panagia N., Van Dyk S. D., Weiler K. W., Sramek R. A., Stockdale C. J., Murata K. P., 2006, ApJ, 646, 369
Pejcha O., Metzger B. D., Tomida K., 2016a, MNRAS, 455, 4351 Pejcha O., Metzger B. D., Tomida K., 2016b, MNRAS, 461, 2527 Piersanti L., Tornambé A., Yungelson L., Straniero O., 2013, in Di Stefano R., Orio M., Moe M., eds, IAU Symposium Vol. 281, Binary Paths to Type Ia Supernovae Explosions. pp 209-212, doi:10.1017/S1743921312015049

Piersanti L., Tornambé A., Yungelson L. R., 2014, MNRAS, 445, 3239

Readhead A. C. S., 1994, ApJ, 426, 51

Ruiter A. J., Belczynski K., Sim S. A., Seitenzahl I. R., Kwiatkowski D., 2014, MNRAS, 440, L101

Rupen M. P., Dhawan V., Mioduszewski A., 2001, IAU Circ., 7717

Rupen M. P., Mioduszewski A. J., Sokoloski J. L., 2008, ApJ, 688,559

Sarbadhicary S. K., Badenes C., Chomiuk L., Caprioli D., Huizenga D., 2017, MNRAS, 464, 2326

Seaquist E., Bode M., 2008, Cambridge Astrophysics Series, 43, 141

Seaquist E. R., Palimaka J., 1977, ApJ, 217, 781

Shen K. J., Bildsten L., 2009, ApJ, 699, 1365

Shepherd M. C., 1997a, in Hunt G., Payne H., eds, Astronomical Society of the Pacific Conference Series Vol. 125, Astronomical Data Analysis Software and Systems VI. p. 77

Shepherd M. C., 1997b, in Hunt G., Payne H., eds, Astronomical Society of the Pacific Conference Series Vol. 125, Astronomical Data Analysis Software and Systems VI. p. 77

Shepherd M. C., Pearson T. J., Taylor G. B., 1994, in BAAS. pp 987-989

Shimamoto S., Sakon I., Onaka T., Usui F., Ootsubo T., Doi Y., Ohsawa R., Ishihara D., 2017, Publication of Korean Astronomical Society, 32, 109

Sokoloski J. L., Rupen M. P., Mioduszewski A. J., 2008, ApJ, 685, L137

Taylor A. R., Seaquist E. R., Hollis J. M., Pottasch S. R., 1987, A\&A, 183, 38

Vlasov A., Vurm I., Metzger B. D., 2016, MNRAS, 463, 394

Weiler K. W., Panagia N., Montes M. J., Sramek R. A., 2002, ARA\&A, 40, 387

Weston J. H. S., et al., 2016a, MNRAS, 457, 887

Weston J. H. S., et al., 2016b, MNRAS, 460, 2687

Woosley S. E., Kasen D., 2011, ApJ, 734, 38

Woudt P. A., Ribeiro V. A., 2014, ASPC, 490

Woudt P. A., et al., 2009, ApJ, 706, 738

Wright A. E., Barlow M. J., 1975, MNRAS, 170, 41

Yaron O., Prialnik D., Shara M. M., Kovetz A., 2005, ApJ, 623, 398

van der Walt S., Colbert S. C., Varoquaux G., 2011, Computing in Science and Engineering, 13, 22

\section{APPENDIX A: SIMPLE MODEL FOR SYNCHROTRON EMISSION FROM NOVAE}

Chevalier (1982) developed a simple formalism for interpreting synchrotron emission from stellar explosions. The model was developed for application to $\mathrm{SNe}$, where the ejecta blast wave crashes into pre-existing circumstellar material. The shock between the ejecta and the circumstellar material accelerates particles to relativistic speeds (through diffusive shock acceleration; e.g., Blandford \& Ostriker 1978; Bell 1978) and amplifies the magnetic field (through the streaming instability; e.g., Bell 2004). The relativistic electrons gyrate along the magnetic field lines to give rise to synchrotron emission. Here we give a few more details of how this model might be applied to V445 Pup.

The magnetic field strength in the synchrotron-emitting 
region is simply $B=\sqrt{8 \pi U_{B}}$. The energy density of relativistic electrons is spread between electrons with a power-law distribution of energies, $d N(E)=N_{0} E^{-p} d E$ where $N_{0}$ is a constant, and we assume a minimum energy of relativistic electrons equivalent to the rest mass energy of the electron (Chevalier 1998). We take $p=2.1$, as measured from the spectrum of V445 Pup ( 44.1$)$, noting that some of the equations in Chevalier (1998) are not defined for $p=2.0$.

To convert from a magnetic field strength to physical parameters of the system, we assume a fraction of the postshock energy density $\left(\rho_{\mathrm{disc}} v_{\mathrm{w}}^{2}\right)$ is converted into amplified magnetic fields and relativistic electrons (as described in $\S 4.3)$. In supernova synchrotron models, conversion factors of $\varepsilon_{e}=\varepsilon_{B}=0.1$ are often assumed (e.g. Chevalier \& Fransson 2006; Chomiuk et al. 2012; Cendes et al. 2020). However, recent considerations imply that $\varepsilon_{B}$ could be much lower $(\lesssim 0.01$, Kundu et al. 2017; Lundqvist et al. 2020), and $\varepsilon_{e}$ may also be lower at slower shock velocities (e.g., Sarbadhicary et al. 2017). To roughly estimate the density of material surrounding V445 Pup, we take $\varepsilon_{e}=\varepsilon_{B}=0.01$.

We estimate $V_{\text {synch }} \approx 10^{47} \mathrm{~cm}^{-3}$, as for a shell that has been expanding for 460 days at $1600 \mathrm{~km} \mathrm{~s}^{-1}$, with a thickness of $10 \%$. If we take a relatively modest flux density for V445 Pup around this time of $3 \mathrm{mJy}$ at an intermediate frequency of $8.4 \mathrm{GHz}$ (consistent with measurements both before and after the day 460 radio flare), this implies that the shock is interacting with material of density, $\sim 3000 \mathrm{~cm}^{-3}$. Given that this interaction is probably with the equatorial disc, our estimate of a spherical $V_{\text {synch }}$ is over-simplistic. The implied density will increase as the volume filling factor of the synchrotron-emitting material decreases.

\section{APPENDIX B: SYNCHROTRON SELF ABSORPTION IN V445 PUP?}

Could synchrotron self-absorption be the cause of the opacity on the rise to the radio peaks? We can use the flux density at radio peak on day $\sim 460$, combined with a few other rough estimates of system parameters, to estimate the opacity due to synchrotron self-absorption $\left(\tau_{\mathrm{SSA}}\right)$. If synchrotron self-absorption is the dominant source of opacity, we would expect $\tau_{\mathrm{SSA}} \approx 1$ at radio peak.

Using the same assumptions described in Appendix A, but instead taking a flux of $12 \mathrm{mJy}$, we estimate $B=0.01$ $\mathrm{G}$. We can then plug these quantities into the equation for synchrotron self-absorption optical depth (Equation 1 of Chevalier 1998), and find that $\tau_{\mathrm{SSA}} \approx 10^{-8}$ at the peak of the day 460 radio flare. As $\tau_{\mathrm{SSA}}$ is eight orders of magnitude smaller than unity, we conclude synchrotron self-absorption is not the dominant source of opacity. We note that for $\tau_{\mathrm{SSA}} \approx 1$, the emitting volume would need to be $V_{\text {synch }} \approx 10^{38}$ $\mathrm{cm}^{-3}$, a factor of a billion smaller than our estimate in Appendix A-but the emitting region is directly constrained by our radio imaging (§3.3), and exclude this possibility.

\section{APPENDIX C: VLA OBSERVATIONS OF V445 PUP, MEASURED FLUX DENSITIES AND SPECTRAL INDICES}

This paper has been typeset from a $\mathrm{T}_{\mathrm{E}} \mathrm{X} / \mathrm{LATEX}$ file prepared by the author. 
Table C1. Log of VLA observations of V445 Pup.

\begin{tabular}{|c|c|c|c|c|c|c|c|c|c|}
\hline \multirow{2}{*}{$\begin{array}{l}\text { Observation } \\
\text { Date }\end{array}$} & \multirow{2}{*}{$\begin{array}{c}t \\
\text { (MJD) }\end{array}$} & \multirow{2}{*}{$\begin{array}{c}t-t_{0} \\
\text { (Days) }\end{array}$} & \multirow[t]{2}{*}{ Configuration } & \multicolumn{6}{|c|}{ Observation time on target (min) } \\
\hline & & & & $1.43 \mathrm{GHz}$ & $4.86 \mathrm{GHz}$ & $8.46 \mathrm{GHz}$ & $14.94 \mathrm{GHz}$ & $22.46 \mathrm{GHz}$ & $43.34 \mathrm{GHz}$ \\
\hline 2001 Oct 04 & 52187 & 337 & $\mathrm{C} \& \mathrm{D}$ & 8.9 & 6.7 & 6.7 & 6.7 & 6.7 & 6.2 \\
\hline 2001 Oct 08 & 52191 & 341 & $C \& D$ & 3.1 & 4.7 & 4.7 & 3.7 & 5.2 & 5.4 \\
\hline 2001 Oct 10 & 52193 & 343 & $C \& D$ & 3.6 & 4.2 & 4.2 & 5.1 & 5.2 & 5.6 \\
\hline 2001 Oct 12 & 52195 & 345 & $\mathrm{D}$ & 2.6 & 4.1 & 4.2 & 5.1 & 5.2 & 5.6 \\
\hline 2001 Oct 14 & 52197 & 347 & $\mathrm{D}$ & 4.4 & 4.2 & 4.2 & 5.7 & 6.9 & 6.4 \\
\hline 2001 Oct 17 & 52200 & 350 & $\mathrm{D}$ & 10.2 & 4.2 & 4.2 & 6.2 & 7.6 & ... \\
\hline 2001 Oct 22 & 52204 & 354 & $\mathrm{D}$ & 2.9 & 2.7 & 2.4 & 5.2 & & 4.7 \\
\hline 2001 Oct 24 & 52207 & 357 & $\mathrm{D}$ & 4.9 & 2.7 & 2.8 & 6.9 & 7.6 & ... \\
\hline 2001 Oct 26 & 52209 & 359 & $\mathrm{D}$ & 5.4 & 5.7 & 7.4 & 7.7 & 7.6 & ... \\
\hline 2001 Oct 27 & 52210 & 360 & $\mathrm{D}$ & 7.3 & 5.6 & 7.4 & 7.7 & 7.7 & ... \\
\hline 2001 Oct 29 & 52212 & 362 & D & 4.4 & 4.7 & 4.7 & 6.9 & 7.4 & ... \\
\hline 2001 Nov 02 & 52216 & 366 & D & 7.6 & 8.4 & 8.6 & 7.6 & 7.7 & ... \\
\hline 2001 Nov 04 & 52218 & 368 & D & 7.8 & 8.4 & 24.6 & 9.2 & $\ldots$ & ... \\
\hline 2001 Nov 06 & 52220 & 370 & $\mathrm{D}$ & 5.9 & 5.3 & 5.2 & 6.2 & $\ldots$ & ... \\
\hline 2001 Nov 07 & 52221 & 371 & $\mathrm{D}$ & 5.9 & 4.7 & 4.2 & 5.8 & 6.6 & ... \\
\hline 2001 Nov 11 & 52224 & 374 & $\mathrm{D}$ & 6.1 & $\ldots$ & 4.2 & 5.3 & 6.2 & 5.7 \\
\hline 2001 Nov 14 & 52227 & 377 & $\mathrm{D}$ & $\ldots$ & 3.9 & 3.7 & $\ldots$ & $\ldots$ & ... \\
\hline 2001 Nov 15 & 52229 & 379 & $\mathrm{D}$ & 4.1 & 4.2 & 2.2 & 5.1 & & ... \\
\hline 2001 Nov 16 & 52230 & 380 & $\mathrm{D}$ & $\ldots$ & 5.2 & 4.9 & 6.2 & 6.9 & ... \\
\hline 2001 Nov 18 & 52231 & 381 & D & 4.2 & 5.1 & 3.1 & 6.2 & 6.9 & ... \\
\hline 2001 Nov 20 & 52233 & 383 & $\mathrm{D}$ & 4.6 & 4.2 & 4.2 & 6.0 & 6.9 & 6.2 \\
\hline 2001 Nov 23 & 52236 & 386 & $\mathrm{D}$ & $\ldots$ & 4.9 & $\ldots$ & $\ldots$ & $\ldots$ & ... \\
\hline 2001 Nov 24 & 52237 & 387 & $\mathrm{D}$ & $\ldots$ & 4.4 & 5.1 & 5.7 & 7.1 & ... \\
\hline 2001 Nov 26 & 52240 & 390 & $\mathrm{D}$ & 8.1 & 4.2 & $\ldots$ & 6.4 & $\ldots$ & ... \\
\hline 2001 Nov 29 & 52242 & 392 & $\mathrm{D}$ & 5.1 & 5.1 & 5.4 & 6.1 & 7.1 & ... \\
\hline 2001 Dec 07 & 52250 & 400 & D & 8.2 & 4.2 & 4.2 & 6.2 & $\ldots$ & ... \\
\hline 2001 Dec 09 & 52252 & 402 & $\mathrm{D}$ & $\ldots$ & $\ldots$ & 5.2 & 6.2 & $\ldots$ & ... \\
\hline 2001 Dec 31 & 52274 & 424 & $\mathrm{D}$ & 5.3 & 4.2 & 4.1 & 6.2 & $\ldots$ & ... \\
\hline 2002 Jan 05 & 52279 & 429 & $\mathrm{D}$ & 7.6 & 4.2 & 4.1 & 6.1 & 6.7 & 6.2 \\
\hline 2002 Jan 12 & 52286 & 436 & D & $\ldots$ & 4.1 & 4.2 & 4.7 & $\ldots$ & ... \\
\hline 2002 Jan 13 & 52287 & 437 & $\mathrm{D}$ & $\ldots$ & 4.1 & $\ldots$ & 5.6 & $\ldots$ & ... \\
\hline 2002 Jan 23 & 52297 & 447 & A & 6.4 & 5.9 & 7.6 & 10.6 & $\ldots$ & ... \\
\hline 2002 Feb 07 & 52312 & 462 & A & 4.2 & 3.2 & 9.1 & 4.7 & $\ldots$ & ... \\
\hline 2002 Feb 14 & 52319 & 469 & A & 1.9 & 4.6 & 10.9 & 4.7 & $\ldots$ & ... \\
\hline 2002 Feb 21 & 52326 & 476 & A & 4.1 & 2.3 & 6.2 & 4.7 & $\ldots$ & ... \\
\hline 2002 Mar 01 & 52334 & 484 & A & 3.9 & 3.1 & 7.6 & 3.7 & $\ldots$ & ... \\
\hline 2002 Mar 06 & 52339 & 489 & A & $\ldots$ & $\ldots$ & 4.7 & $\ldots$ & $\ldots$ & ... \\
\hline 2002 Mar 10 & 52343 & 493 & A & 4.6 & 4.6 & 9.1 & 3.7 & 4.2 & ... \\
\hline 2002 Mar 18 & 52351 & 501 & A & 2.6 & 3.2 & 4.6 & 3.2 & 3.2 & ... \\
\hline 2002 Apr 03 & 52367 & 517 & A & 3.2 & 5.2 & 6.1 & 3.6 & 3.2 & ... \\
\hline 2002 Apr 21 & 52385 & 535 & A & 3.2 & 5.2 & 6.1 & $\ldots$ & $\ldots$ & ... \\
\hline 2002 Apr 29 & 52393 & 543 & A & 6.6 & 5.2 & 15.3 & 5.9 & $\ldots$ & ... \\
\hline 2002 May 04 & 52398 & 548 & A & 6.1 & 5.2 & $\ldots$ & 6.1 & $\cdots$ & ... \\
\hline 2002 May 17 & 52412 & 562 & $A \& B$ & 3.3 & 4.2 & $\ldots$ & $\ldots$ & $\ldots$ & ... \\
\hline 2002 May 26 & 52421 & 571 & $A \& B$ & 3.2 & 3.1 & $\ldots$ & $\ldots$ & $\ldots$ & ... \\
\hline 2002 Jun 07 & 52433 & 583 & $\mathrm{~A} \& \mathrm{~B}$ & 4.0 & 3.2 & $\ldots$ & $\ldots$ & $\ldots$ & ... \\
\hline 2002 Jun 17 & 52443 & 593 & B & 3.6 & 3.2 & $\ldots$ & $\ldots$ & $\ldots$ & ... \\
\hline 2002 Jun 25 & 52451 & 601 & B & 3.4 & 3.2 & 3.1 & .. & $\ldots$ & ... \\
\hline 2002 Jul 05 & 52461 & 611 & B & 3.4 & 3.2 & 3.1 & $\ldots$ & $\ldots$ & ... \\
\hline 2002 Jul 19 & 52475 & 625 & B & $\ldots$ & $\ldots$ & 16.3 & $\ldots$ & $\ldots$ & ... \\
\hline $2002 \mathrm{Jul} 27$ & 52483 & 633 & B & 4.1 & 3.2 & 4.7 & $\ldots$ & $\ldots$ & ... \\
\hline 2002 Aug 17 & 52504 & 654 & B & 4.72 & 6.2 & 10.6 & $\ldots$ & $\ldots$ & ... \\
\hline 2002 Aug 30 & 52517 & 667 & B & 10.6 & 14.0 & 13.6 & $\ldots$ & $\ldots$ & ... \\
\hline 2002 Sep 01 & 52519 & 669 & B & 6.6 & 8.1 & 7.9 & $\ldots$ & $\ldots$ & ... \\
\hline 2002 Oct 04 & 52552 & 702 & B & $\ldots$ & 7.1 & 7.6 & $\ldots$ & $\ldots$ & ... \\
\hline 2002 Oct 28 & 52575 & 725 & B & 4.9 & 4.1 & 4.2 & $\ldots$ & $\ldots$ & ... \\
\hline 2002 Oct 31 & 52579 & 729 & B & $\ldots$ & $\ldots$ & 4.1 & $\ldots$ & $\ldots$ & 4.7 \\
\hline 2002 Dec 09 & 52617 & 767 & C & $\ldots$ & 8.2 & 8.4 & $\ldots$ & $\ldots$ & ... \\
\hline 2002 Dec 21 & 52629 & 779 & C & $\ldots$ & 6.2 & 6.1 & $\ldots$ & $\ldots$ & ... \\
\hline 2002 Dec 29 & 52637 & 787 & $\mathrm{C}$ & $\ldots$ & 6.1 & 6.1 & $\ldots$ & $\ldots$ & ... \\
\hline 2003 Jan 03 & 52642 & 792 & $\mathrm{C}$ & $\ldots$ & 8.1 & 8.1 & $\ldots$ & $\ldots$ & ... \\
\hline 2003 Jan 06 & 52645 & 795 & $\mathrm{C}$ & $\ldots$ & 8.1 & 8.2 & $\ldots$ & $\ldots$ & ... \\
\hline 2003 Jan 08 & 52647 & 797 & $\mathrm{C}$ & $\ldots$ & 8.1 & 8.2 & $\ldots$ & $\ldots$ & ... \\
\hline
\end{tabular}

'...' indicates no observations for this epoch at that frequency 
Table C1 - continued Log of VLA observations of V445 Pup.

\begin{tabular}{|c|c|c|c|c|c|c|c|c|c|}
\hline \multirow{2}{*}{$\begin{array}{l}\text { Observation } \\
\text { Date }\end{array}$} & \multirow{2}{*}{$\begin{array}{c}t \\
\text { (MJD) }\end{array}$} & \multirow{2}{*}{$\begin{array}{c}t-t_{0} \\
\text { (Days) }\end{array}$} & \multirow[t]{2}{*}{ Configuration } & \multicolumn{6}{|c|}{ Observation time on target (min) } \\
\hline & & & & $1.43 \mathrm{GHz}$ & $4.86 \mathrm{GHz}$ & $8.46 \mathrm{GHz}$ & $14.94 \mathrm{GHz}$ & $22.46 \mathrm{GHz}$ & $43.34 \mathrm{GHz}$ \\
\hline 2003 Jan 14 & 52653 & 803 & $\mathrm{C}$ & $\ldots$ & 9.2 & 9.2 & $\ldots$ & $\ldots$ & $\ldots$ \\
\hline 2003 Jan 20 & 52659 & 809 & $\mathrm{C}$ & $\ldots$ & 9.1 & 9.1 & $\ldots$ & $\ldots$ & $\ldots$ \\
\hline 2003 Jan 29 & 52668 & 818 & $\mathrm{C}$ & $\ldots$ & 9.7 & 9.0 & $\ldots$ & $\ldots$ & $\ldots$ \\
\hline 2003 Feb 06 & 52676 & 826 & $\mathrm{D}$ & 8.6 & 9.7 & 9.1 & $\ldots$ & $\ldots$ & $\ldots$ \\
\hline 2003 Feb 23 & 52693 & 843 & $\mathrm{D}$ & $\ldots$ & 9.7 & 9.1 & $\ldots$ & $\ldots$ & $\ldots$ \\
\hline 2003 Mar 04 & 52702 & 852 & $\mathrm{D}$ & $\ldots$ & 10.4 & 9.9 & $\ldots$ & $\ldots$ & $\ldots$ \\
\hline 2003 Mar 11 & 52709 & 859 & $\mathrm{D}$ & $\ldots$ & 10.3 & 12.2 & $\ldots$ & $\ldots$ & $\ldots$ \\
\hline 2003 Mar 13 & 52711 & 861 & $\mathrm{D}$ & $\ldots$ & 16.4 & 22.1 & $\ldots$ & $\ldots$ & $\ldots$ \\
\hline 2003 Mar 19 & 52717 & 867 & $\mathrm{D}$ & $\ldots$ & 10.4 & 9.2 & $\ldots$ & $\ldots$ & $\ldots$ \\
\hline 2003 Mar 26 & 52724 & 874 & $\mathrm{D}$ & $\ldots$ & 10.6 & 9.2 & $\ldots$ & $\ldots$ & $\ldots$ \\
\hline 2003 Apr 18 & 52748 & 898 & $\mathrm{D}$ & $\ldots$ & 15.9 & 12.2 & $\ldots$ & $\ldots$ & $\ldots$ \\
\hline 2003 May 30 & 52790 & 940 & A & 8.8 & 6.9 & 6.2 & $\ldots$ & $\ldots$ & $\ldots$ \\
\hline 2003 Jun 04 & 52795 & 945 & A & 8.6 & 7.0 & $\ldots$ & $\ldots$ & $\ldots$ & $\ldots$ \\
\hline 2003 Jun 16 & 52807 & 957 & A & 8.8 & 7.7 & $\ldots$ & $\ldots$ & $\ldots$ & $\ldots$ \\
\hline 2003 Jun 30 & 52821 & 971 & A & 8.9 & 7.7 & $\ldots$ & $\ldots$ & $\ldots$ & $\ldots$ \\
\hline $2003 \mathrm{Jul} 18$ & 52839 & 989 & A & $\ldots$ & $\ldots$ & 15.2 & $\ldots$ & $\ldots$ & $\ldots$ \\
\hline 2003 Jul 21 & 52842 & 992 & A & $\ldots$ & 15.2 & $\ldots$ & $\ldots$ & $\ldots$ & $\ldots$ \\
\hline 2003 Jul 25 & 52846 & 996 & A & $\ldots$ & 16.2 & $\ldots$ & $\ldots$ & $\ldots$ & $\ldots$ \\
\hline 2003 Jul 31 & 52856 & 1002 & A & $\ldots$ & $\ldots$ & 9.6 & $\ldots$ & $\ldots$ & $\ldots$ \\
\hline 2003 Aug 11 & 52863 & 1013 & A & 8.6 & 9.2 & 11.1 & $\ldots$ & $\ldots$ & $\ldots$ \\
\hline 2003 Aug 25 & 52877 & 1027 & A & 6.9 & 9.1 & 10.7 & $\ldots$ & $\ldots$ & $\ldots$ \\
\hline 2003 Sep 03 & 52886 & 1036 & A & 7.3 & 9.1 & 18.2 & $\ldots$ & $\ldots$ & $\ldots$ \\
\hline 2003 Sep 11 & 52894 & 1044 & A & 10.9 & 10.1 & 22.4 & $\ldots$ & $\ldots$ & $\ldots$ \\
\hline 2003 Sep 19 & 52902 & 1052 & A & 10.9 & $\ldots$ & 24.4 & $\ldots$ & $\ldots$ & $\ldots$ \\
\hline $2003 \mathrm{Sep}$ & 52905 & 1055 & A & 8.6 & 8.1 & 10.7 & $\ldots$ & $\ldots$ & $\ldots$ \\
\hline 2003 Sep 27 & 52910 & 1060 & $A \& B$ & 10.8 & 10.1 & $\ldots$ & $\ldots$ & $\ldots$ & $\ldots$ \\
\hline 2003 Sep 29 & 52912 & 1062 & $A \& B$ & $\ldots$ & 10.6 & 11.6 & $\ldots$ & $\ldots$ & $\ldots$ \\
\hline 2003 Oct 08 & 52921 & 1071 & $A \& B$ & $\ldots$ & 12.2 & $\ldots$ & $\ldots$ & $\ldots$ & $\ldots$ \\
\hline 2003 Oct 21 & 52934 & 1084 & B & $\ldots$ & 10.2 & 14.2 & $\ldots$ & $\ldots$ & $\ldots$ \\
\hline 2003 Nov 02 & 52946 & 1096 & B & $\ldots$ & 8.2 & 8.3 & $\ldots$ & & \\
\hline 003 Nov 09 & 52953 & 1103 & B & $\ldots$ & 8.7 & 18.4 & $\ldots$ & $\ldots$ & $\ldots$ \\
\hline 2003 Nov 18 & 52962 & 1112 & B & $\ldots$ & $\ldots$ & 14.4 & $\ldots$ & $\ldots$ & $\ldots$ \\
\hline 2003 Nov 24 & 52967 & 1117 & B & $\ldots$ & 8.7 & 10.2 & $\ldots$ & $\ldots$ & $\ldots$ \\
\hline 2003 Dec 15 & 52988 & 1138 & B & 5.9 & 9.1 & 12.2 & $\ldots$ & $\ldots$ & $\ldots$ \\
\hline 2003 Dec 28 & 53001 & 1151 & B & 5.9 & 9.1 & 12.2 & $\ldots$ & $\ldots$ & $\ldots$ \\
\hline 2004 Jan 03 & 53007 & 1157 & B & 5.1 & 9.1 & 12.2 & $\ldots$ & $\ldots$ & $\ldots$ \\
\hline 2004 Jan 08 & 53012 & 1162 & B & 5.7 & 9.2 & 11.7 & $\ldots$ & $\ldots$ & $\ldots$ \\
\hline 2004 Jan 14 & 53018 & 1168 & B & $\ldots$ & 9.2 & 11.5 & $\ldots$ & $\ldots$ & $\ldots$ \\
\hline 2004 Jan 23 & 53027 & 1177 & $\mathrm{~B} \& \mathrm{C}$ & $\ldots$ & 6.2 & 6.4 & $\ldots$ & $\ldots$ & $\ldots$ \\
\hline 2004 Feb 01 & 53036 & 1186 & $\mathrm{~B} \& \mathrm{C}$ & 5.9 & 6.2 & 6.2 & & & \\
\hline 2004 Feb 02 & 53037 & 1187 & $B \& C$ & $\ldots$ & $\ldots$ & 4.1 & 10.3 & 10.2 & 10.7 \\
\hline $2004 \mathrm{Feb} 12$ & 53047 & 1197 & $\mathrm{~B} \& \mathrm{C}$ & $\ldots$ & 4.2 & 4.1 & $\ldots$ & 9.0 & 10.7 \\
\hline $2004 \mathrm{Feb} 24$ & 53059 & 1209 & $\mathrm{~B} \& \mathrm{C}$ & $\ldots$ & 4.2 & 4.1 & $\ldots$ & 11.9 & $\ldots$ \\
\hline 2004 Mar 02 & 53066 & 1216 & $\mathrm{~B} \& \mathrm{C}$ & $\ldots$ & 4.2 & 4.1 & $\ldots$ & 12.3 & $\ldots$ \\
\hline 2004 Mar 10 & 53074 & 1224 & $\mathrm{C}$ & $\ldots$ & 57.4 & $\ldots$ & $\ldots$ & $\ldots$ & $\ldots$ \\
\hline 2004 Mar 20 & 53084 & 1234 & $\mathrm{C}$ & $\ldots$ & 4.2 & 8.1 & $\ldots$ & $\ldots$ & $\ldots$ \\
\hline 2004 Mar 22 & 53086 & 1236 & $\mathrm{C}$ & $\ldots$ & $\ldots$ & 6.2 & $\ldots$ & $\ldots$ & $\ldots$ \\
\hline 2004 Apr 01 & 53096 & 1246 & $\mathrm{C}$ & $\ldots$ & $\ldots$ & 8.2 & $\ldots$ & $\ldots$ & $\ldots$ \\
\hline 2004 Apr 07 & 53102 & 1252 & $\mathrm{C}$ & $\ldots$ & 4.2 & 8.1 & $\ldots$ & 13.6 & $\ldots$ \\
\hline 2004 Apr 15 & 53111 & 1261 & $\mathrm{C}$ & $\ldots$ & 4.2 & 4.8 & $\ldots$ & $\ldots$ & $\ldots$ \\
\hline 2004 May 03 & 53129 & 1279 & $\mathrm{C}$ & $\ldots$ & 5.2 & 8.1 & $\ldots$ & $\ldots$ & $\ldots$ \\
\hline 2004 May 10 & 53136 & 1286 & $\mathrm{C}$ & 10.6 & 10.6 & 9.9 & $\ldots$ & $\ldots$ & $\ldots$ \\
\hline 2004 May 17 & 53143 & 1293 & $C \& D$ & 10.6 & 8.9 & 10.1 & $\ldots$ & $\ldots$ & $\ldots$ \\
\hline 2004 May 22 & 53147 & 1297 & $C \& D$ & 7.4 & $\ldots$ & $\ldots$ & $\ldots$ & $\ldots$ & $\ldots$ \\
\hline 2004 May 30 & 53156 & 1306 & $C \& D$ & 6.8 & 5.2 & 5.1 & $\ldots$ & $\ldots$ & $\ldots$ \\
\hline 2004 Jun 12 & 53169 & 1319 & $C \& D$ & 6.0 & 8.9 & 10.1 & $\ldots$ & $\ldots$ & $\ldots$ \\
\hline 2004 Jun 25 & 53182 & 1332 & $\mathrm{D}$ & $\ldots$ & 8.9 & 10.1 & $\ldots$ & $\ldots$ & $\ldots$ \\
\hline 2004 Jul 01 & 53188 & 1338 & $\mathrm{D}$ & $\ldots$ & 8.6 & 10.1 & $\ldots$ & $\ldots$ & $\ldots$ \\
\hline 2004 Jul 10 & 53197 & 1347 & $\mathrm{D}$ & $\ldots$ & $\ldots$ & 10.1 & 20.4 & $\ldots$ & $\ldots$ \\
\hline $2004 \mathrm{Jul} 17$ & 53204 & 1354 & $\mathrm{D}$ & $\ldots$ & 8.9 & 10.1 & 19.2 & $\ldots$ & $\ldots$ \\
\hline $2004 \mathrm{Jul} 23$ & 53210 & 1360 & $\mathrm{D}$ & $\ldots$ & 8.6 & 6.9 & $\ldots$ & $\ldots$ & $\ldots$ \\
\hline 2004 Aug 07 & 53225 & 1375 & $\mathrm{D}$ & $\ldots$ & 8.9 & 10.1 & 12.7 & $\ldots$ & $\ldots$ \\
\hline 2004 Aug 21 & 53239 & 1389 & $\mathrm{D}$ & $\ldots$ & 9.2 & 10.2 & 10.2 & $\ldots$ & $\ldots$ \\
\hline
\end{tabular}

'...' indicates no observations for this epoch at that frequency 
Table C1 - continued Log of VLA observations of V445 Pup.

\begin{tabular}{|c|c|c|c|c|c|c|c|c|c|}
\hline \multirow{2}{*}{$\begin{array}{l}\text { Observation } \\
\text { Date }\end{array}$} & \multirow{2}{*}{$\begin{array}{c}t \\
(\mathrm{MJD})\end{array}$} & \multirow{2}{*}{$\begin{array}{c}t-t_{0} \\
\text { (Days) }\end{array}$} & \multirow[t]{2}{*}{ Configuration } & \multicolumn{6}{|c|}{ Observation time on target (min) } \\
\hline & & & & $1.43 \mathrm{GHz}$ & $4.86 \mathrm{GHz}$ & $8.46 \mathrm{GHz}$ & $14.94 \mathrm{GHz}$ & $22.46 \mathrm{GHz}$ & $43.34 \mathrm{GHz}$ \\
\hline 2004 Sep 09 & 53258 & 1408 & A & $\ldots$ & 9.2 & 10.2 & 10.2 & $\ldots$ & $\ldots$ \\
\hline 2004 Sep 23 & 53272 & 1422 & A & $\ldots$ & 8.6 & 12.2 & $\ldots$ & $\ldots$ & $\ldots$ \\
\hline 2004 Oct 01 & 53280 & 1430 & A & $\ldots$ & $\ldots$ & 18.2 & $\ldots$ & $\ldots$ & $\ldots$ \\
\hline 2004 Oct 08 & 53287 & 1437 & A & $\ldots$ & $\ldots$ & 17.4 & $\ldots$ & $\ldots$ & $\ldots$ \\
\hline 2004 Oct 16 & 53295 & 1445 & A & $\ldots$ & $\ldots$ & 15.2 & $\ldots$ & $\ldots$ & $\ldots$ \\
\hline 2004 Oct 31 & 53310 & 1460 & A & $\ldots$ & 9.1 & 9.2 & $\ldots$ & $\ldots$ & $\ldots$ \\
\hline 2004 Nov 03 & 53312 & 1462 & A & $\ldots$ & 8.2 & 18.2 & $\ldots$ & $\ldots$ & $\ldots$ \\
\hline 2004 Nov 07 & 53317 & 1467 & A & $\ldots$ & $\ldots$ & 14.1 & $\ldots$ & $\ldots$ & $\ldots$ \\
\hline 2004 Nov 12 & 53321 & 1471 & A & $\ldots$ & $\ldots$ & 21.1 & $\ldots$ & $\ldots$ & $\ldots$ \\
\hline 2004 Nov 20 & 53329 & 1479 & A & $\ldots$ & $\ldots$ & 20.8 & $\ldots$ & $\ldots$ & $\ldots$ \\
\hline 2004 Nov 29 & 53338 & 1488 & A & $\ldots$ & $\ldots$ & 21.2 & $\ldots$ & $\ldots$ & $\ldots$ \\
\hline 2004 Dec 22 & 53361 & 1511 & A & $\ldots$ & $\ldots$ & 14.6 & $\ldots$ & $\ldots$ & $\ldots$ \\
\hline 2004 Dec 31 & 53370 & 1520 & A & $\ldots$ & $\ldots$ & 15.2 & $\ldots$ & $\ldots$ & $\ldots$ \\
\hline 2005 Jan 06 & 53376 & 1526 & A & $\ldots$ & $\ldots$ & 13.6 & $\ldots$ & $\ldots$ & $\ldots$ \\
\hline 2005 Jan 16 & 53386 & 1536 & $A \& B$ & $\ldots$ & $\ldots$ & 10.2 & $\ldots$ & $\ldots$ & $\ldots$ \\
\hline 2005 Jan 24 & 53394 & 1544 & $A \& B$ & $\ldots$ & $\ldots$ & 8.6 & $\ldots$ & $\ldots$ & $\ldots$ \\
\hline 2005 Jan 29 & 53399 & 1549 & $A \& B$ & $\ldots$ & 12.1 & 11.8 & $\ldots$ & $\ldots$ & $\ldots$ \\
\hline 2005 Feb 03 & 53404 & 1554 & $A \& B$ & $\ldots$ & $\ldots$ & 7.1 & $\ldots$ & $\ldots$ & $\ldots$ \\
\hline 2005 Feb 26 & 53427 & 1577 & B & $\ldots$ & 8.6 & 9.2 & $\ldots$ & $\ldots$ & $\ldots$ \\
\hline 2005 Mar 13 & 53442 & 1592 & B & 8.9 & 8.1 & 10.3 & $\ldots$ & $\ldots$ & $\ldots$ \\
\hline 2005 Mar 20 & 53449 & 1599 & B & 8.8 & 8.1 & 10.4 & $\ldots$ & $\ldots$ & $\ldots$ \\
\hline 2005 Mar 25 & 53454 & 1604 & B & 8.9 & 8.1 & 10.2 & $\ldots$ & $\ldots$ & $\ldots$ \\
\hline 2005 Apr 04 & 53464 & 1614 & B & $\ldots$ & 37.7 & $\ldots$ & $\ldots$ & $\ldots$ & $\ldots$ \\
\hline 2005 Apr 14 & 53475 & 1625 & B & $\ldots$ & 6.6 & 8.2 & $\ldots$ & $\ldots$ & $\ldots$ \\
\hline 2005 Apr 26 & 53486 & 1636 & B & $\ldots$ & 6.4 & 9.1 & $\ldots$ & $\ldots$ & $\ldots$ \\
\hline 2005 May 06 & 53497 & 1647 & B & $\ldots$ & 5.2 & 9.0 & $\ldots$ & $\ldots$ & $\ldots$ \\
\hline 2005 Мay 14 & 53504 & 1654 & B & $\ldots$ & 5.1 & 10.2 & $\ldots$ & $\ldots$ & $\ldots$ \\
\hline 2005 May 29 & 53520 & 1670 & B & $\ldots$ & 22.4 & $\ldots$ & $\ldots$ & $\ldots$ & $\ldots$ \\
\hline 2005 Jun 03 & 53525 & 1675 & B & $\ldots$ & 19.9 & $\ldots$ & $\ldots$ & $\ldots$ & $\ldots$ \\
\hline 2005 Jun 12 & 53534 & 1684 & $\mathrm{~B} \& \mathrm{C}$ & $\ldots$ & $\ldots$ & 10.7 & $\cdots$ & $\ldots$ & ... \\
\hline 2005 Jun 18 & 53540 & 1690 & $\mathrm{~B} \& \mathrm{C}$ & $\ldots$ & $\ldots$ & 15.4 & $\ldots$ & $\ldots$ & $\ldots$ \\
\hline 2005 Jun 23 & 53545 & 1695 & $\mathrm{~B} \& \mathrm{C}$ & $\ldots$ & 13.1 & 12.2 & $\ldots$ & $\ldots$ & $\ldots$ \\
\hline 2005 Jul 02 & 53554 & 1704 & $\mathrm{~B} \& \mathrm{C}$ & 11.4 & 13.1 & 12.2 & $\ldots$ & $\ldots$ & $\ldots$ \\
\hline 2005 Jul 09 & 53561 & 1711 & $\mathrm{C}$ & $\ldots$ & $\ldots$ & 4.2 & $\ldots$ & $\ldots$ & $\ldots$ \\
\hline 2005 Jul 14 & 53566 & 1716 & $\mathrm{C}$ & $\ldots$ & 10.9 & $\ldots$ & $\ldots$ & $\ldots$ & $\ldots$ \\
\hline 2005 Jul 23 & 53575 & 1725 & $\mathrm{C}$ & $\ldots$ & 10.1 & 14.2 & $\ldots$ & $\ldots$ & $\ldots$ \\
\hline 2005 Aug 02 & 53585 & 1735 & $\mathrm{C}$ & $\ldots$ & $\ldots$ & 17.2 & $\ldots$ & $\ldots$ & $\ldots$ \\
\hline 2005 Aug 06 & 53589 & 1739 & $\mathrm{C}$ & $\ldots$ & $\ldots$ & 6.2 & $\ldots$ & $\ldots$ & $\ldots$ \\
\hline 2005 Aug 27 & 53610 & 1760 & $\mathrm{C}$ & $\ldots$ & 20.2 & 12.2 & $\ldots$ & $\ldots$ & $\ldots$ \\
\hline 2005 Sep 05 & 53619 & 1769 & $\mathrm{C}$ & $\ldots$ & 20.4 & 16.7 & $\ldots$ & $\ldots$ & $\ldots$ \\
\hline 2005 Sep 25 & 53639 & 1789 & $\mathrm{C}$ & $\ldots$ & 22.2 & $\ldots$ & $\ldots$ & $\ldots$ & $\ldots$ \\
\hline 2005 Sep 27 & 53641 & 1791 & $\mathrm{C}$ & $\ldots$ & 12.2 & 18.2 & $\ldots$ & $\ldots$ & $\ldots$ \\
\hline 2005 Oct 01 & 53645 & 1795 & $\mathrm{C}$ & $\ldots$ & 20.4 & 16.7 & $\ldots$ & $\ldots$ & $\ldots$ \\
\hline 2005 Oct 05 & 53649 & 1799 & C\&D & $\ldots$ & $\ldots$ & 14.4 & $\ldots$ & $\ldots$ & $\ldots$ \\
\hline 2005 Oct 16 & 53660 & 1810 & C\&D & $\ldots$ & $\ldots$ & 10.4 & $\ldots$ & 18.7 & ... \\
\hline 2005 Oct 23 & 53666 & 1816 & C\&D & $\ldots$ & 61.6 & 14.2 & $\ldots$ & $\ldots$ & $\ldots$ \\
\hline 2005 Oct 31 & 53675 & 1825 & $C \& D$ & $\ldots$ & $\ldots$ & 18.4 & $\ldots$ & $\ldots$ & $\ldots$ \\
\hline 2005 Nov 08 & 53683 & 1833 & D & $\ldots$ & 9.1 & 9.2 & $\ldots$ & $\ldots$ & $\ldots$ \\
\hline 2005 Nov 15 & 53690 & 1840 & D & $\ldots$ & $\ldots$ & 9.2 & $\ldots$ & $\ldots$ & $\ldots$ \\
\hline 2005 Nov 21 & 53696 & 1846 & $\mathrm{D}$ & $\ldots$ & $\ldots$ & 21.2 & $\ldots$ & $\ldots$ & $\ldots$ \\
\hline 2005 Nov 29 & 53703 & 1853 & $\mathrm{D}$ & $\ldots$ & $\ldots$ & 17.4 & $\ldots$ & $\ldots$ & $\ldots$ \\
\hline 2005 Dec 01 & 53705 & 1855 & D & $\ldots$ & 13.2 & 15.6 & $\ldots$ & $\ldots$ & $\ldots$ \\
\hline 2005 Dec 11 & 53715 & 1865 & $\mathrm{D}$ & $\ldots$ & 13.1 & 15.6 & $\ldots$ & $\ldots$ & $\cdots$ \\
\hline 2005 Dec 20 & 53724 & 1874 & $\mathrm{D}$ & $\ldots$ & 13.1 & 15.2 & $\ldots$ & $\ldots$ & $\ldots$ \\
\hline 2006 Jan 13 & 53748 & 1898 & $\mathrm{D}$ & $\ldots$ & $\ldots$ & 14.9 & $\ldots$ & $\ldots$ & $\ldots$ \\
\hline 2006 Jan 15 & 53750 & 1900 & $\mathrm{D}$ & $\ldots$ & $\ldots$ & 18.2 & $\ldots$ & $\ldots$ & $\ldots$ \\
\hline 2006 Jan 22 & 53757 & 1907 & $\mathrm{D}$ & $\ldots$ & 9.2 & $\ldots$ & $\ldots$ & $\ldots$ & $\ldots$ \\
\hline 2006 Feb 09 & 53775 & 1925 & $\mathrm{~A}$ & $\ldots$ & $\ldots$ & 16.2 & $\ldots$ & $\ldots$ & ... \\
\hline 2006 Mar 15 & 53809 & 1959 & $\mathrm{~A}$ & 9.6 & $\ldots$ & 12.2 & $\ldots$ & $\ldots$ & ... \\
\hline 2006 Mar 22 & 53816 & 1966 & A & 9.5 & $\ldots$ & $\ldots$ & $\ldots$ & $\cdots$ & ... \\
\hline 2006 April 07 & 53832 & 1982 & $\mathrm{~A}$ & 8.9 & $\ldots$ & $\ldots$ & $\ldots$ & $\ldots$ & $\ldots$ \\
\hline 2006 May 08 & 53863 & 2013 & A & $\ldots$ & $\ldots$ & 20.2 & $\ldots$ & $\ldots$ & ... \\
\hline 2006 May 10 & 53865 & 2015 & A & $\ldots$ & $\ldots$ & 14.2 & $\ldots$ & $\ldots$ & $\ldots$ \\
\hline
\end{tabular}

'...' indicates no observations for this epoch at that frequency 
18 Nyamai et al.

Table C1 - continued Log of VLA observations of V445 Pup.

\begin{tabular}{|c|c|c|c|c|c|c|c|c|c|}
\hline \multirow{2}{*}{$\begin{array}{l}\text { Observation } \\
\text { Date }\end{array}$} & \multirow{2}{*}{$\begin{array}{c}t \\
(\mathrm{MJD})\end{array}$} & \multirow{2}{*}{$\begin{array}{c}t-t_{0} \\
\text { (Days) }\end{array}$} & \multirow[t]{2}{*}{ Configuration } & \multicolumn{6}{|c|}{ Observation time on target (min) } \\
\hline & & & & $1.43 \mathrm{GHz}$ & $4.86 \mathrm{GHz}$ & $8.46 \mathrm{GHz}$ & $14.94 \mathrm{GHz}$ & $22.46 \mathrm{GHz}$ & $43.34 \mathrm{GHz}$ \\
\hline 2006 Aug 05 & 53953 & 2103 & B & $\ldots$ & .. & 24.6 & .. & $\ldots$ & $\ldots$ \\
\hline 2006 Aug 09 & 53957 & 2107 & B & $\ldots$ & 24.4 & $\ldots$ & $\ldots$ & $\ldots$ & $\ldots$ \\
\hline 2006 Aug 31 & 53979 & 2129 & B & $\ldots$ & 20.6 & $\ldots$ & $\ldots$ & $\ldots$ & $\ldots$ \\
\hline 2006 Sep 11 & 53990 & 2140 & B & $\ldots$ & 38.3 & $\ldots$ & $\ldots$ & $\ldots$ & $\ldots$ \\
\hline 2007 Jan 23 & 54123 & 2273 & $C \& D$ & $\ldots$ & $\ldots$ & 29.3 & $\ldots$ & $\ldots$ & $\ldots$ \\
\hline 2007 Sep 30 & 54374 & 2524 & $\mathrm{~A} \& \mathrm{~B}$ & $\ldots$ & $\ldots$ & 36.0 & $\ldots$ & $\ldots$ & $\ldots$ \\
\hline 2008 Jan 17 & 54482 & 2632 & B & $\ldots$ & $\ldots$ & 28.0 & $\ldots$ & $\ldots$ & $\ldots$ \\
\hline 2008 Mar 28 & 54554 & 2704 & $\mathrm{C}$ & $\ldots$ & $\ldots$ & 10.4 & $\ldots$ & $\ldots$ & $\ldots$ \\
\hline
\end{tabular}

'...' indicates no observations for this epoch at that frequency 
Table C2. Flux densities and spectral indices of V445 Pup

\begin{tabular}{|c|c|c|c|c|c|c|c|c|}
\hline \multirow{2}{*}{$\begin{array}{l}t \\
\text { (MJD) }\end{array}$} & \multirow{2}{*}{$\begin{array}{c}t-t_{0} \\
\text { (Days) }\end{array}$} & \multicolumn{6}{|c|}{ Radio flux densities (mJy) } & \multirow[t]{2}{*}{$\alpha$} \\
\hline & & $1.43 \mathrm{GHz}$ & $4.86 \mathrm{GHz}$ & $8.46 \mathrm{GHz}$ & $14.94 \mathrm{GHz}$ & $22.46 \mathrm{GHz}$ & $43.34 \mathrm{GHz}$ & \\
\hline 52187 & 337 & $12.62 \pm 0.67$ & $13.85 \pm 0.71$ & $12.01 \pm 0.62$ & $8.69 \pm 0.91$ & $5.98 \pm 0.88$ & $5.10 \pm 1.21$ & $-0.46 \pm 0.08$ \\
\hline 52191 & 341 & $14.26 \pm 0.76$ & $14.01 \pm 0.73$ & $11.33 \pm 0.60$ & $7.54 \pm 1.17$ & $8.61 \pm 1.26$ & $4.30 \pm 1.18$ & $-0.43 \pm 0.07$ \\
\hline 52193 & 343 & $13.54 \pm 0.80$ & $12.48 \pm 0.66$ & $9.29 \pm 0.52$ & $7.27 \pm 0.88$ & $5.13 \pm 1.05$ & $2.49 \pm 0.78$ & $-0.58 \pm 0.06$ \\
\hline 52195 & 345 & $12.15 \pm 0.85$ & $11.46 \pm 0.63$ & $8.67 \pm 0.49$ & $5.83 \pm 0.71$ & $5.66 \pm 0.75$ & $7.00 \pm 1.22$ & $-0.24 \pm 0.07$ \\
\hline 52197 & 347 & $14.75 \pm 1.21$ & $10.4 \pm 0.57$ & $7.64 \pm 0.42$ & $7.93 \pm 0.87$ & $7.79 \pm 0.92$ & $7.21 \pm 1.16$ & $-0.25 \pm 0.06$ \\
\hline 52200 & 350 & $13.96 \pm 1.18$ & $8.81 \pm 0.50$ & $8.45 \pm 0.46$ & $8.31 \pm 0.91$ & $7.36 \pm 1.0$ & $\ldots$ & $-0.23 \pm 0.06$ \\
\hline 52204 & 354 & $10.30 \pm 1.31$ & $8.83 \pm 0.79$ & $7.56 \pm 0.46$ & $5.82 \pm 0.66$ & $\ldots$ & $3.14 \pm 0.86$ & $-0.27 \pm 0.07$ \\
\hline 52207 & 357 & $10.20 \pm 0.93$ & $7.37 \pm 0.41$ & $7.30 \pm 0.44$ & $6.18 \pm 0.68$ & $4.2 \pm 0.57$ & $\ldots$ & $-0.24 \pm 0.02$ \\
\hline 52209 & 359 & $12.5 \pm 1.18$ & $8.2 \pm 0.44$ & $7.17 \pm 0.37$ & $5.69 \pm 0.60$ & $5.64 \pm 0.72$ & $\ldots$ & $-0.30 \pm 0.02$ \\
\hline 52210 & 360 & $9.42 \pm 0.84$ & $7.23 \pm 0.40$ & $6.72 \pm 0.36$ & $4.52 \pm 0.58$ & $3.71 \pm 0.69$ & $\ldots$ & $-0.26 \pm 0.07$ \\
\hline 52212 & 362 & $8.42 \pm 0.45$ & $7.52 \pm 0.41$ & $6.62 \pm 0.38$ & $4.30 \pm 0.51$ & $3.94 \pm 0.72$ & $\ldots$ & $-0.20 \pm 0.07$ \\
\hline 52216 & 366 & $8.14 \pm 0.44$ & $6.99 \pm 0.37$ & $5.29 \pm 0.30$ & $3.62 \pm 0.50$ & $1.89 \pm 0.41$ & $\ldots$ & $-0.65 \pm 0.13$ \\
\hline 52218 & 368 & $8.29 \pm 0.42$ & $6.33 \pm 0.35$ & $4.75 \pm 0.25$ & $3.95 \pm 0.52$ & $\ldots$ & $\ldots$ & $-0.30 \pm 0.05$ \\
\hline 52220 & 370 & $10.26 \pm 0.61$ & $5.66 \pm 0.35$ & $4.28 \pm 0.26$ & $3.16 \pm 0.49$ & $\ldots$ & $\ldots$ & $-0.49 \pm 0.01$ \\
\hline 52221 & 371 & $8.56 \pm 0.45$ & $5.52 \pm 0.33$ & $4.28 \pm 0.26$ & $3.51 \pm 0.40$ & $2.53 \pm 0.54$ & $\ldots$ & $-0.39 \pm 0.02$ \\
\hline 52224 & 374 & $8.39 \pm 0.42$ & $\ldots$ & $4.48 \pm 0.28$ & $4.57 \pm 0.65$ & $2.96 \pm 0.77$ & $2.89 \pm 0.477$ & $-0.32 \pm 0.03$ \\
\hline 52227 & 377 & & $\ldots$ & $4.81 \pm 0.037$ & $4.29 \pm 0.29$ & $\ldots$ & $\ldots$ & $\ldots$ \\
\hline 52229 & 379 & $6.79 \pm 0.93$ & $4.68 \pm 0.29$ & $4.71 \pm 0.35$ & $4.89 \pm 0.83$ & $\ldots$ & $\ldots$ & $-0.15 \pm 0.09$ \\
\hline 52230 & 380 & $\ldots$ & $4.5 \pm 0.28$ & $5.01 \pm 0.29$ & $4.80 \pm 0.60$ & $3.72 \pm 0.64$ & $\ldots$ & $-0.22 \pm 0.13$ \\
\hline 52231 & 381 & $5.61 \pm 0.81$ & $4.81 \pm 0.30$ & $5.25 \pm 0.29$ & $4.38 \pm 0.54$ & $3.88 \pm 0.59$ & $\ldots$ & $-0.10 \pm 0.06$ \\
\hline 52233 & 383 & $9.99 \pm 0.58$ & $5.10 \pm 0.33$ & $4.66 \pm 0.26$ & $3.65 \pm 0.47$ & $3.15 \pm 0.48$ & $2.14 \pm 0.63$ & $-0.43 \pm 0.04$ \\
\hline 52236 & 386 & $\ldots$ & $4.95 \pm 0.41$ & $\ldots$ & $\ldots$ & $\ldots$ & $\ldots$ & $\ldots$ \\
\hline 52237 & 387 & $\ldots$ & $4.49 \pm 0.33$ & $4.01 \pm 0.26$ & $3.15 \pm 0.44$ & $2.39 \pm 0.52$ & $\ldots$ & $-0.34 \pm 0.08$ \\
\hline 52240 & 390 & $6.39 \pm 0.37$ & $4.51 \pm 0.32$ & $\ldots$ & $2.35 \pm 0.51$ & $\ldots$ & $\ldots$ & $-0.33 \pm 0.08$ \\
\hline 52242 & 392 & $12.38 \pm 1.08$ & $4.10 \pm 0.29$ & $3.46 \pm 0.20$ & $2.30 \pm 0.33$ & $2.59 \pm 0.72$ & $\ldots$ & $-0.43 \pm 0.03$ \\
\hline 52250 & 400 & $4.91 \pm 0.47$ & $3.30 \pm 0.30$ & $2.49 \pm 0.26$ & $1.97 \pm 0.47$ & $\ldots$ & $\ldots$ & $-0.38 \pm 0.03$ \\
\hline 52252 & 402 & $\ldots$ & $\ldots$ & $2.16 \pm 0.22$ & $2.30 \pm 0.40$ & $\ldots$ & $\ldots$ & $\ldots$ \\
\hline 52274 & 424 & $5.03 \pm 0.27$ & $2.02 \pm 0.23$ & $1.71 \pm 0.18$ & $3.09 \pm 0.71$ & $\ldots$ & $\ldots$ & $-0.54 \pm 0.16$ \\
\hline 52279 & 429 & $6.31 \pm 0.57$ & $2.02 \pm 0.17$ & $2.65 \pm 0.19$ & $3.12 \pm 0.42$ & $3.77 \pm 0.57$ & $3.20 \pm 1.15$ & $0.36 \pm 0.06$ \\
\hline 52286 & 436 & $\ldots$ & $3.91 \pm 0.33$ & $4.67 \pm 0.33$ & $4.36 \pm 0.78$ & $\ldots$ & $\ldots$ & $0.19 \pm 0.15$ \\
\hline 52287 & 437 & $\ldots$ & $3.61 \pm 0.30$ & $\ldots$ & $2.80 \pm 0.62$ & $\ldots$ & $\ldots$ & $\ldots$ \\
\hline 52297 & 447 & $4.46 \pm 0.29$ & $4.93 \pm 0.31$ & $9.38 \pm 0.52$ & $14.01 \pm 1.52$ & $\ldots$ & $\ldots$ & $0.44 \pm 0.18$ \\
\hline 52312 & 462 & $6.91 \pm 0.36$ & $21.92 \pm 1.12$ & $17.68 \pm 0.89$ & $11.39 \pm 1.30$ & $\ldots$ & $\ldots$ & $-0.51 \pm 0.13$ \\
\hline 52319 & 469 & $11.49 \pm 0.63$ & $15.53 \pm 0.83$ & $13.02 \pm 0.67$ & $4.35 \pm 0.95$ & $\ldots$ & $\ldots$ & $-0.54 \pm 0.44$ \\
\hline 52326 & 476 & $16.27 \pm 0.83$ & $14.09 \pm 0.74$ & $10.38 \pm 0.54$ & $6.46 \pm 1.04$ & $\ldots$ & $\ldots$ & $-0.26 \pm 0.09$ \\
\hline 52334 & 484 & $17.91 \pm 1.12$ & $13.34 \pm 0.82$ & $9.23 \pm 0.50$ & $6.02 \pm 0.91$ & $\ldots$ & $\ldots$ & $-0.39 \pm 0.08$ \\
\hline 52339 & 489 & $\ldots$ & $\ldots$ & $8.53 \pm 0.57$ & $\ldots$ & $\ldots$ & $\ldots$ & $\ldots$ \\
\hline 52343 & 493 & $18.65 \pm 0.95$ & $11.48 \pm 0.63$ & $8.44 \pm 0.45$ & $4.73 \pm 0.60$ & $3.39 \pm 0.42$ & $\ldots$ & $-0.53 \pm 0.08$ \\
\hline 52351 & 501 & $16.44 \pm 0.84$ & $9.03 \pm 0.86$ & $6.72 \pm 0.43$ & $4.22 \pm 1.00$ & $2.93 \pm 0.57$ & $\ldots$ & $-0.53 \pm 0.04$ \\
\hline 52367 & 517 & $12.30 \pm 0.68$ & $7.31 \pm 0.40$ & $6.15 \pm 0.34$ & $4.66 \pm 0.55$ & $3.12 \pm 0.96$ & $\ldots$ & $-0.41 \pm 0.02$ \\
\hline 52385 & 535 & $9.30 \pm 0.49$ & $5.25 \pm 0.41$ & $3.66 \pm 0.28$ & $\ldots$ & $\ldots$ & $\ldots$ & $-0.51 \pm 0.04$ \\
\hline 52393 & 543 & $7.91 \pm 0.41$ & $4.53 \pm 0.30$ & $2.88 \pm 0.18$ & $2.01 \pm 0.35$ & $\ldots$ & $\ldots$ & $-0.55 \pm 0.05$ \\
\hline 52398 & 548 & $6.52 \pm 0.35$ & $3.69 \pm 0.29$ & $\ldots$ & $1.70 \pm 0.32$ & $\ldots$ & $\ldots$ & $-0.51 \pm 0.06$ \\
\hline 52412 & 562 & $7.66 \pm 0.59$ & $1.97 \pm 0.13$ & $\ldots$ & $\ldots$ & $\ldots$ & $\ldots$ & $\ldots$ \\
\hline 52421 & 571 & $4.84 \pm 0.31$ & $1.95 \pm 0.16$ & $\ldots$ & $\ldots$ & $\ldots$ & $\ldots$ & ... \\
\hline 52433 & 583 & $2.32 \pm 0.13$ & $1.43 \pm 0.09$ & $\ldots$ & $\ldots$ & $\ldots$ & $\ldots$ & ... \\
\hline 52443 & 593 & $2.79 \pm 0.16$ & $1.10 \pm 0.13$ & $\ldots$ & $\ldots$ & $\ldots$ & $\ldots$ & $\ldots$ \\
\hline 52451 & 601 & $2.10 \pm 0.11$ & $1.40 \pm 0.14$ & $1.07 \pm 0.26$ & $\ldots$ & $\ldots$ & $\ldots$ & $-0.35 \pm 0.02$ \\
\hline 52461 & 611 & $3.35 \pm 0.21$ & $1.01 \pm 0.12$ & $0.94 \pm 0.20$ & $\ldots$ & $\ldots$ & $\ldots$ & $-0.87 \pm 0.15$ \\
\hline 52475 & 625 & $\ldots$ & $\ldots$ & $0.68 \pm 0.08$ & $\ldots$ & $\ldots$ & $\ldots$ & $\ldots$ \\
\hline 52483 & 633 & $1.89 \pm 0.11$ & $1.02 \pm 0.11$ & $0.88 \pm 0.14$ & $\ldots$ & $\ldots$ & $\ldots$ & $-0.46 \pm 0.04$ \\
\hline 52504 & 654 & $1.73 \pm 0.15$ & $0.99 \pm 0.15$ & $0.75 \pm 0.10$ & $\ldots$ & $\ldots$ & $\ldots$ & $-0.47 \pm 0.01$ \\
\hline 52517 & 667 & $1.06 \pm 0.08$ & $0.61 \pm 0.06$ & $0.49 \pm 0.05$ & $\ldots$ & $\ldots$ & $\ldots$ & $-0.44 \pm 0.01$ \\
\hline 52519 & 669 & $2.09 \pm 0.11$ & $1.20 \pm 0.15$ & $0.92 \pm 0.17$ & $\ldots$ & $\ldots$ & $\ldots$ & $-0.46 \pm 0.00$ \\
\hline 52552 & 702 & $\ldots$ & $0.78 \pm 0.12$ & $0.61 \pm 0.11$ & $\ldots$ & $\ldots$ & $\ldots$ & $\ldots$ \\
\hline 52575 & 725 & $1.46 \pm 0.19$ & $0.89 \pm 0.07$ & $0.49 \pm 0.11$ & $\ldots$ & $\ldots$ & $\ldots$ & $-0.47 \pm 0.07$ \\
\hline 52579 & 729 & $\ldots$ & $\ldots$ & $0.52 \pm 0.12$ & $\ldots$ & $\ldots$ & $1.50 \pm 0.31$ & $\ldots$ \\
\hline 52617 & 767 & $\cdots$ & $1.15 \pm 0.08$ & $0.98 \pm 0.14$ & $\ldots$ & $\ldots$ & $\ldots$ & ... \\
\hline 52629 & 779 & $\ldots$ & $0.80 \pm 0.06$ & $0.86 \pm 0.12$ & $\ldots$ & $\ldots$ & $\ldots$ & ... \\
\hline 52637 & 787 & $\ldots$ & $0.99 \pm 0.11$ & $0.88 \pm 0.14$ & $\ldots$ & $\ldots$ & $\ldots$ & ... \\
\hline 52642 & 792 & $\ldots$ & $0.85 \pm 0.11$ & $0.71 \pm 0.10$ & $\ldots$ & $\ldots$ & $\ldots$ & $\ldots$ \\
\hline 52645 & 795 & $\cdots$ & $1.08 \pm 0.12$ & $0.84 \pm 0.10$ & $\cdots$ & $\ldots$ & $\ldots$ & ... \\
\hline 52647 & 797 & $\ldots$ & $0.77 \pm 0.11$ & $0.66 \pm 0.16$ & $\ldots$ & $\ldots$ & $\ldots$ & $\ldots$ \\
\hline
\end{tabular}

'...' indicates no measurements for flux density for this epoch at that frequency 
Table C2 - continued Flux densities and spectral indices of V445 Pup

\begin{tabular}{|c|c|c|c|c|c|c|c|c|}
\hline \multirow{2}{*}{$\begin{array}{l}t \\
(\mathrm{MJD})\end{array}$} & \multirow{2}{*}{$\begin{array}{c}t-t_{0} \\
\text { (Days) }\end{array}$} & \multirow[b]{2}{*}{$1.43 \mathrm{GHz}$} & \multicolumn{3}{|c|}{ Radio flux densities (mJy) } & \multirow[b]{2}{*}{$22.46 \mathrm{GHz}$} & \multirow[b]{2}{*}{$43.34 \mathrm{GHz}$} & \multirow[t]{2}{*}{$\alpha$} \\
\hline & & & $4.86 \mathrm{GHz}$ & $8.46 \mathrm{GHz}$ & $14.94 \mathrm{GHz}$ & & & \\
\hline 52653 & 803 & $\ldots$ & $0.84 \pm 0.10$ & $0.61 \pm 0.10$ & $\ldots$ & $\ldots$ & $\ldots$ & ... \\
\hline 52659 & 809 & $\ldots$ & $0.77 \pm 0.07$ & $0.65 \pm 0.10$ & $\ldots$ & $\ldots$ & $\ldots$ & $\ldots$ \\
\hline 52668 & 818 & $\ldots$ & $1.22 \pm 0.09$ & $0.60 \pm 0.07$ & $\ldots$ & $\ldots$ & $\ldots$ & $\ldots$ \\
\hline 52676 & 826 & $2.85 \pm 0.59$ & $0.78 \pm 0.09$ & $0.77 \pm 0.07$ & $\ldots$ & $\ldots$ & $\ldots$ & $-0.79 \pm 0.08$ \\
\hline 52693 & 843 & $\ldots$ & $0.86 \pm 0.11$ & $0.63 \pm 0.09$ & $\ldots$ & $\ldots$ & $\ldots$ & $\ldots$ \\
\hline 52702 & 852 & $\ldots$ & $0.90 \pm 0.11$ & $0.724 \pm 0.13$ & $\ldots$ & $\ldots$ & $\ldots$ & $\ldots$ \\
\hline 52709 & 859 & $\ldots$ & $0.93 \pm 0.10$ & $0.80 \pm 0.12$ & $\ldots$ & $\ldots$ & $\ldots$ & $\ldots$ \\
\hline 52711 & 861 & $\ldots$ & $0.94 \pm 0.09$ & $0.74 \pm 0.09$ & $\ldots$ & $\ldots$ & $\ldots$ & $\ldots$ \\
\hline 52717 & 867 & $\ldots$ & $0.84 \pm 0.09$ & $0.83 \pm 0.10$ & $\ldots$ & $\ldots$ & $\ldots$ & $\ldots$ \\
\hline 52724 & 874 & $\cdots$ & $1.42 \pm 0.18$ & $1.15 \pm 0.13$ & $\ldots$ & $\ldots$ & $\ldots$ & $\ldots$ \\
\hline 52748 & 898 & $\ldots$ & $1.17 \pm 0.11$ & $0.66 \pm 0.09$ & $\ldots$ & $\ldots$ & $\ldots$ & $\ldots$ \\
\hline 52790 & 940 & $2.21 \pm 0.19$ & $0.91 \pm 0.09$ & $0.24 \pm 0.04$ & $\ldots$ & $\ldots$ & $\ldots$ & $-0.93 \pm 0.47$ \\
\hline 52795 & 945 & $2.53 \pm 0.27$ & $0.78 \pm 0.08$ & $\ldots$ & $\ldots$ & $\ldots$ & $\ldots$ & $\ldots$ \\
\hline 52807 & 957 & $2.55 \pm 0.24$ & $0.90 \pm 0.10$ & $\ldots$ & $\ldots$ & $\ldots$ & $\ldots$ & $\ldots$ \\
\hline 52821 & 971 & $2.49 \pm 0.22$ & $1.76 \pm 0.22$ & $\ldots$ & $\cdots$ & $\ldots$ & $\ldots$ & $\ldots$ \\
\hline 52839 & 989 & $\ldots$ & $\ldots$ & $1.14 \pm 0.10$ & $\ldots$ & $\ldots$ & $\ldots$ & $\ldots$ \\
\hline 52842 & 992 & $\ldots$ & $1.7 \pm 0.15$ & $\ldots$ & $\ldots$ & $\ldots$ & $\ldots$ & $\ldots$ \\
\hline 52846 & 996 & $\ldots$ & $1.56 \pm 0.15$ & $\ldots$ & $\ldots$ & $\ldots$ & $\ldots$ & $\ldots$ \\
\hline 52852 & 1002 & $\ldots$ & $\ldots$ & $0.65 \pm 0.08$ & $\ldots$ & $\ldots$ & $\ldots$ & .. \\
\hline 52863 & 1013 & $1.69 \pm 0.16$ & $1.37 \pm 0.10$ & $1.07 \pm 0.08$ & $\ldots$ & $\ldots$ & $\ldots$ & $-0.25 \pm 0.08$ \\
\hline 52877 & 1027 & $2.22 \pm 0.32$ & $1.59 \pm 0.23$ & $1.49 \pm 0.20$ & $\ldots$ & $\ldots$ & $\ldots$ & $\ldots$ \\
\hline 52886 & 1036 & $2.67 \pm 0.24$ & $1.89 \pm 0.31$ & $1.01 \pm 0.08$ & $\ldots$ & $\ldots$ & $\cdots$ & $\ldots$ \\
\hline 52894 & 1044 & $1.49 \pm 0.17$ & $1.43 \pm 0.16$ & $0.87 \pm 0.08$ & $\ldots$ & $\ldots$ & $\ldots$ & $\ldots$ \\
\hline 52902 & 1052 & $1.02 \pm 0.16$ & $\ldots$ & $1.10 \pm 0.07$ & $\ldots$ & $\ldots$ & $\ldots$ & $\ldots$ \\
\hline 52905 & 1055 & $1.49 \pm 0.16$ & $1.51 \pm 0.16$ & $1.00 \pm 0.14$ & $\ldots$ & $\ldots$ & $\ldots$ & $\ldots$ \\
\hline 52910 & 1060 & $2.05 \pm 0.18$ & $1.44 \pm 0.12$ & $\ldots$ & $\ldots$ & $\ldots$ & $\ldots$ & $\ldots$ \\
\hline 52912 & 1062 & $\ldots$ & $1.58 \pm 0.20$ & $0.87 \pm 0.08$ & $\ldots$ & $\ldots$ & $\ldots$ & $\ldots$ \\
\hline 52921 & 1071 & $\ldots$ & $1.09 \pm 0.21$ & $\ldots$ & $\ldots$ & $\ldots$ & $\ldots$ & $\ldots$ \\
\hline 52934 & 1084 & $\ldots$ & $1.38 \pm 0.11$ & $1.28 \pm 0.13$ & $\ldots$ & $\ldots$ & $\ldots$ & $\ldots$ \\
\hline 52946 & 1096 & $\ldots$ & $1.32 \pm 0.16$ & $1.28 \pm 0.17$ & $\ldots$ & $\ldots$ & $\ldots$ & $\ldots$ \\
\hline 52953 & 1103 & $\ldots$ & $1.34 \pm 0.16$ & $1.28 \pm 0.12$ & $\ldots$ & $\ldots$ & $\ldots$ & $\ldots$ \\
\hline 52962 & 1112 & $\ldots$ & $\ldots$ & $1.44 \pm 0.15$ & $\ldots$ & $\ldots$ & $\ldots$ & $\ldots$ \\
\hline 52967 & 1117 & $\ldots$ & $1.59 \pm 0.15$ & $1.30 \pm 0.15$ & $\ldots$ & $\ldots$ & $\ldots$ & $\ldots$ \\
\hline 52988 & 1138 & $1.41 \pm 0.25$ & $1.82 \pm 0.11$ & $1.46 \pm 0.08$ & $\ldots$ & $\ldots$ & $\ldots$ & $-0.10 \pm 0.24$ \\
\hline 53001 & 1151 & $2.06 \pm 0.10$ & $1.72 \pm 0.12$ & $1.60 \pm 0.15$ & $\ldots$ & $\ldots$ & $\ldots$ & $\ldots$ \\
\hline 53007 & 1157 & $3.06 \pm 0.22$ & $1.80 \pm 0.18$ & $1.71 \pm 0.18$ & $\ldots$ & $\ldots$ & $\ldots$ & $\ldots$ \\
\hline 53012 & 1162 & $2.01 \pm 0.11$ & $1.90 \pm 0.20$ & $1.62 \pm 0.16$ & ... & $\ldots$ & $\ldots$ & $\ldots$ \\
\hline 53018 & 1168 & $\ldots$ & $1.76 \pm 0.13$ & $1.56 \pm 0.14$ & $\ldots$ & $\ldots$ & $\ldots$ & $\ldots$ \\
\hline 53027 & 1177 & $\ldots$ & $1.74 \pm 0.14$ & $1.67 \pm 0.15$ & $\ldots$ & $\ldots$ & $\ldots$ & $\ldots$ \\
\hline 53036 & 1186 & $2.17 \pm 0.19$ & $2.10 \pm 0.21$ & $1.50 \pm 0.16$ & $\ldots$ & $\ldots$ & $\ldots$ & $\ldots$ \\
\hline 53037 & 1187 & $\ldots$ & $\ldots$ & $1.54 \pm 0.15$ & $1.33 \pm 0.38$ & $1.05 \pm 0.22$ & $0.78 \pm 0.18$ & $-0.42 \pm 0.08$ \\
\hline 53047 & 1197 & $\ldots$ & $2.09 \pm 0.30$ & $1.85 \pm 0.19$ & $\ldots$ & $0.84 \pm 0.17$ & $1.29 \pm 0.37$ & $\ldots$ \\
\hline 53059 & 1209 & $\ldots$ & $1.53 \pm 0.13$ & $1.35 \pm 0.12$ & $\ldots$ & $1.14 \pm 0.27$ & $\ldots$ & $-0.20 \pm 0.02$ \\
\hline 53066 & 1216 & $\cdots$ & $1.33 \pm 0.12$ & $1.30 \pm 0.10$ & $\ldots$ & $0.84 \pm 0.18$ & $\ldots$ & $\ldots$ \\
\hline 53074 & 1224 & $\ldots$ & $1.72 \pm 0.10$ & $\ldots$ & $\ldots$ & $\ldots$ & $\ldots$ & $\ldots$ \\
\hline 53084 & 1234 & $\ldots$ & $1.54 \pm 0.17$ & $1.34 \pm 0.13$ & $\ldots$ & $\ldots$ & $\ldots$ & $\ldots$ \\
\hline 53086 & 1236 & $\ldots$ & $\ldots$ & $1.29 \pm 0.15$ & $\ldots$ & $\ldots$ & $\ldots$ & $\ldots$ \\
\hline 53096 & 1246 & $\ldots$ & $\ldots$ & $1.10 \pm 0.12$ & $\ldots$ & $\ldots$ & $\ldots$ & $\ldots$ \\
\hline 53102 & 1252 & $\ldots$ & $1.15 \pm 0.12$ & $1.27 \pm 0.12$ & $\ldots$ & $0.73 \pm 0.14$ & $\ldots$ & $-0.24 \pm 0.28$ \\
\hline 53111 & 1261 & $\ldots$ & $1.37 \pm 0.20$ & $1.04 \pm 0.12$ & $\ldots$ & $\ldots$ & $\ldots$ & $\ldots$ \\
\hline 53129 & 1279 & $\ldots$ & $1.61 \pm 0.20$ & $1.14 \pm 0.15$ & $\ldots$ & $\ldots$ & $\ldots$ & $\ldots$ \\
\hline 53136 & 1286 & $2.27 \pm 0.28$ & $1.48 \pm 0.10$ & $1.18 \pm 0.08$ & $\ldots$ & $\ldots$ & $\ldots$ & $-0.37 \pm 0.02$ \\
\hline 53143 & 1293 & $0.94 \pm 0.17$ & $1.63 \pm 0.20$ & $1.13 \pm 0.16$ & $\ldots$ & $\ldots$ & $\ldots$ & $\ldots$ \\
\hline 53147 & 1297 & $3.45 \pm 0.50$ & $\ldots$ & $\ldots$ & $\ldots$ & $\ldots$ & $\ldots$ & $\ldots$ \\
\hline 53156 & 1306 & $1.49 \pm 0.18$ & $1.79 \pm 0.22$ & $1.52 \pm 0.18$ & $\ldots$ & $\cdots$ & $\ldots$ & $\ldots$ \\
\hline 53169 & 1319 & $2.51 \pm 0.24$ & $1.50 \pm 0.16$ & $1.23 \pm 0.12$ & $\cdots$ & $\ldots$ & $\cdots$ & $\ldots$ \\
\hline 53182 & 1332 & $\ldots$ & $1.01 \pm 0.10$ & $0.72 \pm 0.08$ & $\ldots$ & $\ldots$ & $\ldots$ & $\ldots$ \\
\hline 53188 & 1338 & $\ldots$ & $1.29 \pm 0.18$ & $0.96 \pm 0.13$ & $\ldots$ & $\ldots$ & $\ldots$ & $\ldots$ \\
\hline 53197 & 1347 & $\ldots$ & $\ldots$ & $0.89 \pm 0.12$ & $0.59 \pm 0.11$ & $\ldots$ & $\ldots$ & $\ldots$ \\
\hline 53204 & 1354 & $\ldots$ & $1.46 \pm 0.12$ & $1.17 \pm 0.09$ & $0.91 \pm 0.19$ & $\ldots$ & $\ldots$ & $-0.42 \pm 0.01$ \\
\hline 53210 & 1360 & $\ldots$ & $1.42 \pm 0.18$ & $1.25 \pm 0.17$ & $\ldots$ & $\ldots$ & $\ldots$ & $\ldots$ \\
\hline 53225 & 1375 & $\ldots$ & $1.21 \pm 0.16$ & $1.07 \pm 0.12$ & $0.98 \pm 0.21$ & $\ldots$ & $\ldots$ & $\ldots$ \\
\hline
\end{tabular}

'...' indicates no measurements for flux density for this epoch at that frequency 
Table C2 - continued Flux densities and spectral indices of V445 Pup

\begin{tabular}{|c|c|c|c|c|c|c|c|c|}
\hline \multirow{2}{*}{$\begin{array}{l}t \\
(\mathrm{MJD})\end{array}$} & \multirow{2}{*}{$\begin{array}{c}t-t_{0} \\
\text { (Days) }\end{array}$} & \multicolumn{6}{|c|}{ Radio flux densities (mJy) } & \multirow[t]{2}{*}{$\alpha$} \\
\hline & & $1.43 \mathrm{GHz}$ & $4.86 \mathrm{GHz}$ & $8.46 \mathrm{GHz}$ & $14.94 \mathrm{GHz}$ & $22.46 \mathrm{GHz}$ & $43.34 \mathrm{GHz}$ & \\
\hline 53239 & 1389 & $\ldots$ & $1.61 \pm 0.18$ & $1.37 \pm 0.15$ & $1.46 \pm 0.28$ & $\ldots$ & $\ldots$ & \\
\hline 53258 & 1408 & $\ldots$ & $1.26 \pm 0.19$ & $1.45 \pm 0.31$ & $1.16 \pm 0.28$ & $\ldots$ & $\ldots$ & $-0.03 \pm 0.17$ \\
\hline 53272 & 1422 & $\ldots$ & $1.07 \pm 0.11$ & $1.43 \pm 0.19$ & $\cdots$ & $\ldots$ & $\ldots$ & $\ldots$ \\
\hline 53280 & 1430 & $\ldots$ & $\ldots$ & $1.51 \pm 0.19$ & $\ldots$ & $\ldots$ & $\ldots$ & $\ldots$ \\
\hline 53287 & 1437 & $\ldots$ & $\ldots$ & $1.56 \pm 0.14$ & $\ldots$ & $\ldots$ & $\ldots$ & $\ldots$ \\
\hline 53295 & 1445 & $\ldots$ & $\ldots$ & $1.71 \pm 0.15$ & $\ldots$ & $\ldots$ & $\ldots$ & $\ldots$ \\
\hline 53310 & 1460 & $\ldots$ & $1.61 \pm 0.14$ & $1.09 \pm 0.16$ & $\ldots$ & $\ldots$ & $\ldots$ & $\ldots$ \\
\hline 53312 & 1462 & $\ldots$ & $1.12 \pm 0.12$ & $1.38 \pm 0.14$ & $\ldots$ & $\ldots$ & $\ldots$ & $\ldots$ \\
\hline 53317 & 1467 & $\ldots$ & $\ldots$ & $1.39 \pm 0.13$ & $\ldots$ & $\ldots$ & $\ldots$ & $\ldots$ \\
\hline 53321 & 1471 & $\ldots$ & $\ldots$ & $1.54 \pm 0.13$ & $\ldots$ & $\ldots$ & $\ldots$ & $\ldots$ \\
\hline 53329 & 1479 & $\ldots$ & $\ldots$ & $1.21 \pm 0.15$ & $\ldots$ & $\ldots$ & $\ldots$ & $\ldots$ \\
\hline 53338 & 1488 & $\ldots$ & $\ldots$ & $1.26 \pm 0.17$ & $\ldots$ & $\ldots$ & $\ldots$ & $\ldots$ \\
\hline 53361 & 1511 & $\ldots$ & $\ldots$ & $1.27 \pm 0.19$ & $\ldots$ & $\ldots$ & $\ldots$ & $\ldots$ \\
\hline 53370 & 1520 & $\ldots$ & $\ldots$ & $1.46 \pm 0.19$ & $\ldots$ & $\ldots$ & $\ldots$ & $\ldots$ \\
\hline 53376 & 1526 & $\ldots$ & $\ldots$ & $1.27 \pm 0.11$ & $\ldots$ & $\ldots$ & $\ldots$ & .. \\
\hline 53386 & 1536 & $\ldots$ & $\ldots$ & $1.21 \pm 0.17$ & $\ldots$ & $\ldots$ & $\ldots$ & $\ldots$ \\
\hline 53394 & 1544 & $\ldots$ & $\ldots$ & $1.38 \pm 0.15$ & $\ldots$ & $\ldots$ & $\ldots$ & $\ldots$ \\
\hline 53399 & 1549 & $\ldots$ & $1.36 \pm 0.15$ & $1.23 \pm 0.14$ & $\ldots$ & $\ldots$ & $\ldots$ & $\ldots$ \\
\hline 53404 & 1554 & $\ldots$ & $\ldots$ & $1.41 \pm 0.16$ & $\ldots$ & $\ldots$ & $\ldots$ & $\ldots$ \\
\hline 53427 & 1577 & $\ldots$ & $1.28 \pm 0.14$ & $1.20 \pm 0.13$ & $\ldots$ & $\ldots$ & $\ldots$ & $\ldots$ \\
\hline 53442 & 1592 & $1.15 \pm 0.12$ & $0.94 \pm 0.07$ & $0.87 \pm 0.08$ & $\ldots$ & $\ldots$ & $\ldots$ & $-0.16 \pm 0.01$ \\
\hline 53449 & 1599 & $0.89 \pm 0.08$ & $1.05 \pm 0.08$ & $1.06 \pm 0.12$ & $\ldots$ & $\ldots$ & $\ldots$ & $\ldots$ \\
\hline 53454 & 1604 & $1.10 \pm 0.11$ & $1.32 \pm 0.18$ & $1.02 \pm 0.16$ & $\ldots$ & $\ldots$ & $\ldots$ & $\ldots$ \\
\hline 53464 & 1614 & $\ldots$ & $1.17 \pm 0.09$ & $\ldots$ & $\ldots$ & $\ldots$ & $\ldots$ & $\ldots$ \\
\hline 53475 & 1625 & $\ldots$ & $0.96 \pm 0.12$ & $0.86 \pm 0.12$ & $\ldots$ & $\ldots$ & $\ldots$ & $\ldots$ \\
\hline 53486 & 1636 & $\ldots$ & $1.21 \pm 0.15$ & $0.93 \pm 0.11$ & $\ldots$ & $\ldots$ & $\ldots$ & $\ldots$ \\
\hline 53497 & 1647 & $\ldots$ & $1.13 \pm 0.14$ & $0.72 \pm 0.10$ & $\ldots$ & $\ldots$ & $\ldots$ & $\cdots$ \\
\hline 53504 & 1654 & $\ldots$ & $0.90 \pm 0.16$ & $0.80 \pm 0.12$ & $\ldots$ & $\ldots$ & $\ldots$ & $\ldots$ \\
\hline 53520 & 1670 & $\ldots$ & $0.94 \pm 0.12$ & $\ldots$ & $\ldots$ & $\ldots$ & $\ldots$ & $\ldots$ \\
\hline 53525 & 1675 & $\ldots$ & $0.99 \pm 0.12$ & $\ldots$ & $\ldots$ & $\ldots$ & $\ldots$ & $\ldots$ \\
\hline 53534 & 1684 & $\ldots$ & $\ldots$ & $0.62 \pm 0.074$ & $\ldots$ & $\ldots$ & $\ldots$ & $\ldots$ \\
\hline 53540 & 1690 & $\ldots$ & $\ldots$ & $0.83 \pm 0.09$ & $\ldots$ & $\ldots$ & $\cdots$ & $\cdots$ \\
\hline 53545 & 1695 & $\ldots$ & $1.01 \pm 0.13$ & $0.76 \pm 0.13$ & $\ldots$ & $\ldots$ & $\ldots$ & $\ldots$ \\
\hline 53554 & 1704 & $1.42 \pm 0.10$ & $0.73 \pm 0.07$ & $0.81 \pm 0.11$ & $\ldots$ & $\ldots$ & $\ldots$ & $\ldots$ \\
\hline 53561 & 1711 & $\ldots$ & $\ldots$ & $0.64 \pm 0.08$ & $\ldots$ & $\ldots$ & $\ldots$ & $\ldots$ \\
\hline 53566 & 1716 & $\ldots$ & $0.77 \pm 0.08$ & $\ldots$ & $\ldots$ & $\ldots$ & $\ldots$ & $\ldots$ \\
\hline 53575 & 1725 & $\ldots$ & $0.68 \pm 0.08$ & $0.51 \pm 0.06$ & $\ldots$ & $\ldots$ & $\ldots$ & $\ldots$ \\
\hline 53585 & 1735 & $\ldots$ & $\ldots$ & $0.47 \pm 0.08$ & $\ldots$ & $\ldots$ & $\ldots$ & $\ldots$ \\
\hline 53589 & 1739 & $\ldots$ & $\ldots$ & $0.78 \pm 0.09$ & $\ldots$ & $\ldots$ & $\ldots$ & $\ldots$ \\
\hline 53610 & 1760 & $\ldots$ & $0.86 \pm 0.09$ & $0.53 \pm 0.07$ & $\ldots$ & $\ldots$ & $\ldots$ & $\ldots$ \\
\hline 53619 & 1769 & $\ldots$ & $1.12 \pm 0.14$ & $0.53 \pm 0.05$ & $\ldots$ & $\ldots$ & $\ldots$ & $\ldots$ \\
\hline 53639 & 1789 & $\ldots$ & $0.73 \pm 0.08$ & $\ldots$ & $\ldots$ & $\ldots$ & $\ldots$ & $\ldots$ \\
\hline 53641 & 1791 & $\ldots$ & $0.58 \pm 0.05$ & $0.68 \pm 0.04$ & $\ldots$ & $\ldots$ & $\ldots$ & $\ldots$ \\
\hline 53645 & 1795 & $\ldots$ & $0.65 \pm 0.05$ & $0.54 \pm 0.05$ & $\ldots$ & $\ldots$ & $\ldots$ & $\ldots$ \\
\hline 53649 & 1799 & $\ldots$ & $\ldots$ & $0.61 \pm 0.09$ & $\ldots$ & $\ldots$ & $\ldots$ & $\ldots$ \\
\hline 53660 & 1810 & $\ldots$ & $\cdots$ & $0.75 \pm 0.07$ & $\cdots$ & $1.10 \pm 0.30$ & $\cdots$ & $\cdots$ \\
\hline 53666 & 1816 & $\ldots$ & $\cdots$ & $0.66 \pm 0.06$ & $\cdots$ & $\ldots$ & $\ldots$ & $\ldots$ \\
\hline 53675 & 1825 & $\ldots$ & $\ldots$ & $0.64 \pm 0.07$ & $\ldots$ & $\ldots$ & $\ldots$ & $\ldots$ \\
\hline 53683 & 1833 & $\ldots$ & $0.62 \pm 0.11$ & $0.58 \pm 0.09$ & $\ldots$ & $\ldots$ & $\ldots$ & $\ldots$ \\
\hline 53689 & 1840 & $\ldots$ & $\ldots$ & $0.57 \pm 0.08$ & $\ldots$ & $\ldots$ & $\ldots$ & $\ldots$ \\
\hline 53696 & 1846 & $\ldots$ & $\ldots$ & $0.54 \pm 0.07$ & $\ldots$ & $\ldots$ & $\ldots$ & $\ldots$ \\
\hline 53703 & 1853 & $\ldots$ & $\ldots$ & $0.74 \pm 0.09$ & $\ldots$ & $\ldots$ & $\ldots$ & $\ldots$ \\
\hline 53705 & 1855 & $\ldots$ & $0.51 \pm 0.05$ & $0.55 \pm 0.05$ & $\ldots$ & $\ldots$ & $\ldots$ & $\ldots$ \\
\hline 53715 & 1865 & $\ldots$ & $0.82 \pm 0.18$ & $0.64 \pm 0.08$ & $\ldots$ & $\ldots$ & $\ldots$ & $\ldots$ \\
\hline 53724 & 1874 & $\ldots$ & $0.60 \pm 0.12$ & $0.53 \pm 0.08$ & $\ldots$ & $\ldots$ & $\ldots$ & $\ldots$ \\
\hline 53748 & 1898 & $\ldots$ & $\cdots$ & $0.59 \pm 0.08$ & $\ldots$ & $\ldots$ & $\ldots$ & $\cdots$ \\
\hline 53750 & 1900 & $\ldots$ & $\ldots$ & $0.48 \pm 0.08$ & $\ldots$ & $\ldots$ & $\ldots$ & $\ldots$ \\
\hline 53757 & 1907 & $\ldots$ & $0.47 \pm 0.09$ & $\ldots$ & $\ldots$ & $\ldots$ & $\ldots$ & $\ldots$ \\
\hline 53775 & 1925 & $\ldots$ & $\ldots$ & $0.43 \pm 0.09$ & $\ldots$ & $\ldots$ & $\ldots$ & $\cdots$ \\
\hline 53809 & 1959 & $0.54 \pm 0.18$ & $\ldots$ & $0.40 \pm 0.09$ & $\cdots$ & $\ldots$ & $\ldots$ & $\cdots$ \\
\hline 53816 & 1966 & $0.51 \pm 0.12$ & $\ldots$ & $\ldots$ & $\ldots$ & $\ldots$ & $\ldots$ & $\ldots$ \\
\hline 53832 & 1982 & $0.61 \pm 0.22$ & $\ldots$ & $\ldots$ & $\ldots$ & $\ldots$ & $\ldots$ & $\ldots$ \\
\hline 53863 & 2013 & $\ldots$ & $\ldots$ & $0.66 \pm 0.09$ & $\ldots$ & $\ldots$ & $\ldots$ & $\ldots$ \\
\hline
\end{tabular}

'...' indicates no measurements for flux density for this epoch at that frequency 
$22 \quad$ Nyamai et al.

Table C2 - continued Flux densities and spectral indices of V445 Pup

\begin{tabular}{|c|c|c|c|c|c|c|c|c|}
\hline \multirow{2}{*}{${ }^{t}(\mathrm{MJD})$} & \multirow{2}{*}{$\begin{array}{c}t-t_{0} \\
\text { (Days) }\end{array}$} & \multicolumn{6}{|c|}{ Radio flux densities (mJy) } & \multirow[t]{2}{*}{$\alpha$} \\
\hline & & $1.43 \mathrm{GHz}$ & $4.86 \mathrm{GHz}$ & $8.46 \mathrm{GHz}$ & $14.94 \mathrm{GHz}$ & $22.46 \mathrm{GHz}$ & $43.34 \mathrm{GHz}$ & \\
\hline 53865 & 2015 & $\ldots$ & $\ldots$ & $0.37 \pm 0.05$ & $\ldots$ & $\ldots$ & $\ldots$ & $\ldots$ \\
\hline 53953 & 2103 & $\ldots$ & $\ldots$ & $0.21 \pm 0.03$ & $\ldots$ & $\ldots$ & $\ldots$ & $\ldots$ \\
\hline 53957 & 2107 & $\ldots$ & $0.42 \pm 0.08$ & $\ldots$ & $\ldots$ & $\ldots$ & $\ldots$ & $\ldots$ \\
\hline 53979 & 2129 & $\ldots$ & $0.30 \pm 0.06$ & $\ldots$ & $\ldots$ & $\ldots$ & $\cdots$ & ... \\
\hline 54123 & 2273 & $\ldots$ & $\ldots$ & $0.29 \pm 0.06$ & $\ldots$ & $\ldots$ & $\ldots$ & $\ldots$ \\
\hline 54374 & 2524 & $\ldots$ & $\ldots$ & $0.22 \pm 0.06$ & $\ldots$ & $\ldots$ & $\ldots$ & $\ldots$ \\
\hline 54482 & 2632 & $\ldots$ & $\cdots$ & $0.26 \pm 0.09$ & $\ldots$ & $\ldots$ & $\cdots$ & ... \\
\hline 54554 & 2704 & $\ldots$ & $\ldots$ & $0.19 \pm 0.04$ & $\ldots$ & $\ldots$ & $\ldots$ & $\ldots$ \\
\hline
\end{tabular}

'...' indicates no measurements for flux density for this epoch at that frequency 